\title{
The ACTC ellerate initiative: large-scale combinatorial cloning of novel human embryonic stem cell derivatives
}

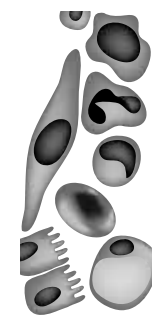

\author{
Michael D West ${ }^{1,2+*}$, \\ R Geoffrey Sargent ${ }^{3}$, \\ Jeff Long'*, \\ Colleen Brown ${ }^{3}$, \\ Jing Song $\mathrm{Chu}^{3}$, \\ Steven Kessier ${ }^{3 \dagger}$, \\ Nikita Derugin 4 , \\ Janani Sampathkumar2, \\ Courtney Burrows ${ }^{3}$, \\ H omayoun Vaziri ${ }^{5}$, \\ Roy Williams ${ }^{6}$, \\ Karen B C hapman ${ }^{3}$, \\ D avid Larocca ${ }^{3}$, \\ Jeanne F Loring ${ }^{6}$ \& \\ James Murai ${ }^{3}$
}

${ }^{\dagger}$ Authors for correspondence ${ }^{1}$ BioTime, Inc, Alameda, CA 94502, USA E-mail: mwes@ biotimemail.com ${ }^{2} \mathrm{U}$ niversity of California, Berkeley, CA 94720, U SA ${ }^{3}$ Advanced Cell Technology, Alameda, CA 94502, U SA E-mail: skesser@ advancedcell.com; skessler@jps.net ${ }^{4} U$ niversity of $C$ alifornia, San Francisco, CA 94143, USA ${ }^{5} 0$ ntario $C$ ancer Institute, Toronto, M 5G 2M 9, Canada ${ }^{6} \mathrm{~T}$ he Burnham Institute for M edical Research, La Jolla, CA 92037, USA *All work by M D West and J Long in relation to this article was carried out at Advanced Cell Technology, CA, USA.

\footnotetext{
Keymords cell culture, cloning, differentiation, embryonic stem cells, gene expression, microarray, telomeres

future medicine $^{\text {prosg }}$
}

\begin{abstract}
Human embryonic stem cells offer a scalable and renewable source of all somatic cell types. Human embryonic progenitor (hEP) cells are partially differentiated endodermal, mesodermal and ectodermal cell types that have not undergone terminal differentiation and express an embryonic pattern of gene expression. Here, we describe a large-scale and reproducible method of isolating a diverse library of clonally purified hEP cell lines, many of which are capable of extended propagation in vitro. Initial microarray and non-negative matrix factorization gene-expression profiling suggests that the library consists of at least 140 distinct clones and contains many previously uncharacterized cell types derived from all germ layers that display diverse embryo- and site-specific homeobox gene expression. Despite the expression of many oncofetal genes, none of the hEP cell lines tested led to tumor formation when transplanted into immunocompromised mice. All hEP lines studied appear to have a finite replicative lifespan but have longer telomeres than most fetal- or adult-derived cells, thereby facilitating their use in the manufacture of purified lineages for research and human therapy.
\end{abstract}

H uman embryonic stem (hES) cells have significant promise for medical research and cell-based therapy owing to their pluripotency [1,2] and presumed ability to cascade through the entire catalog of human embryonic progenitor (hEP) cell types. Embryonic progenitors are cells capable of proliferation and differentiation into one or more terminally differentiated cell types while typically expressing transcripts unique to embryonic stages of development. Embryonic progenitors are therefore usually present during the embryonic stages of development only. Examples of hEP cells include: migrating neural crest [3], early ectodermal progenitors of the cerebellum [4], endodermal progenitors, such as those of the primordial liver [5], and mesodermal precursors of hematopoietic lineages [6]. The isolation and culture of hEP cell lines, although largely unexplored, would facilitate the molecular characterization of these cell types and allow more precise studies of the cellular interactions that occur during the development of human tissues. Thus, there is a need for a general method of isolating hEP cell lines to a level of purity useful in basic research and for the manufacturing of such cells for therapeutic application.

The differentiation of hES cells in vitro is not well understood and current directed differentiation protocols rely heavily on factors previously identified to be necessary for specific aspects of mouse embryonic development in vivo. Accordingly, current protocols employ a strategy wherein hES cells are expanded and exposed to specific differentiation conditions, after which the desired differentiated cell types are purified utilizing affinity-based methods. Since few such purification strategies have been perfected, current differentiation protocols are very inefficient, resulting in heterogeneous populations of differentiated cells wherein the desired cell type represents only a few percent of the population [7]. There are two major concerns with this strategy from a practical standpoint. First, therapeutic applications require a sufficiently pure formation to insure safety (i.e., minimal risk of contaminating cells proliferating to cause tumors or migrating and adversely affecting normal tissue function) [8]. Second, therapeutic applications require a robust and economical scale-up protocol. hES cells are among the most difficult of cells to propagate en masse [9] without losing pluripotency or normal karyotype. Therefore, there is a need for improved methods to increase purity and scalability of hEP cell types.

Early efforts in cell purification from fetal or adult tissues included attempts at purifying cells by clonal isolation. W hile frequently employed in purifying immortalized cells or cells well acclimated to in vitro culture; such as fetal fibroblasts [10], clonal isolation of most normal human cell types often fails either because suitable culture conditions cannot be identified or because the reduced telomere lengths of most fetal, neonatal and adult cell types results in 
replicative senescence before a clonal line can be obtained. While mouse cells generally possess longer telomeres and labile telomerase expression, few tissues even from relatively early in embryonic development, such as E11.5-E13 mouse embryos, are capable of generating stable cell lines and less than $1 \%$ of those can be clonally expanded [West $M D$, Unpublished $D$ ata]. We reasoned, however, that hES-derived hEPs might not have the same limitations as a result of their long initial telomere length and the potential to capture cells at stages of differentiation even earlier than that corresponding to E11.5 mouse cells. In addition, since homologous cells display a surprising degree of spatial diversity owing to site-specific homeobox expression [11] that plays an important role in embryonic pattern formation [12], clonal isolates have the potential to lead to lines with a more uniform pattern of differentiated gene expression. $\mathrm{H}$ ere, we demonstrate the successful derivation of a library of hEP cell lines using a novel two-step isolation method that selects clonal cell populations from hES cells grown and differentiated under a large variety of culture conditions. M any of the hEP lines may represent intermediates of human embryonic differentiation that have not previously been identified or characterized. This establishment of a library of clonal hEP cell lines provides a novel and scalable source of cells for regenerative therapies and provides the first initial characterization of cell types that proliferate relatively well and are therefore likely present in many cultures of embryonic stem (ES)-derived cells.

\section{Results}

Multiplex generation $\&$ characterization of hEP cell clones

In a 'shotgun' strategy to search for hEP cell types capable of propagation in vitro, we implemented a two-step multiplex cell line isolation protocol, designated ACTC ellerate, to identify differentiated hES-derived cell types capable of clonal propagation in an array of differentiation and propagation conditions. In the first step, hES cells (WAO9 [H 9] and M A03) were differentiated under an array of in vitro conditions that included colony in situ differentiation, differentiation as embryoid bodies (EBs), on nonadherent plastic or hanging drops, differentiation in the presence of different growth factors, and for various periods of time (specific differentiation conditions are described in the methods section and the conditions for each cell line are shown in supplementary TableAz). The resultant matrix of cultures are designated 'candidate cultures' (CCS) as shown in Figre1A These CC lines are heterogeneous in nature, although owing to the specific conditions employed in their differentiation, they are enriched in particular cell types

\section{Figure 1. Tw o-step multiplex human embryonic progenitor derivation protocol.}
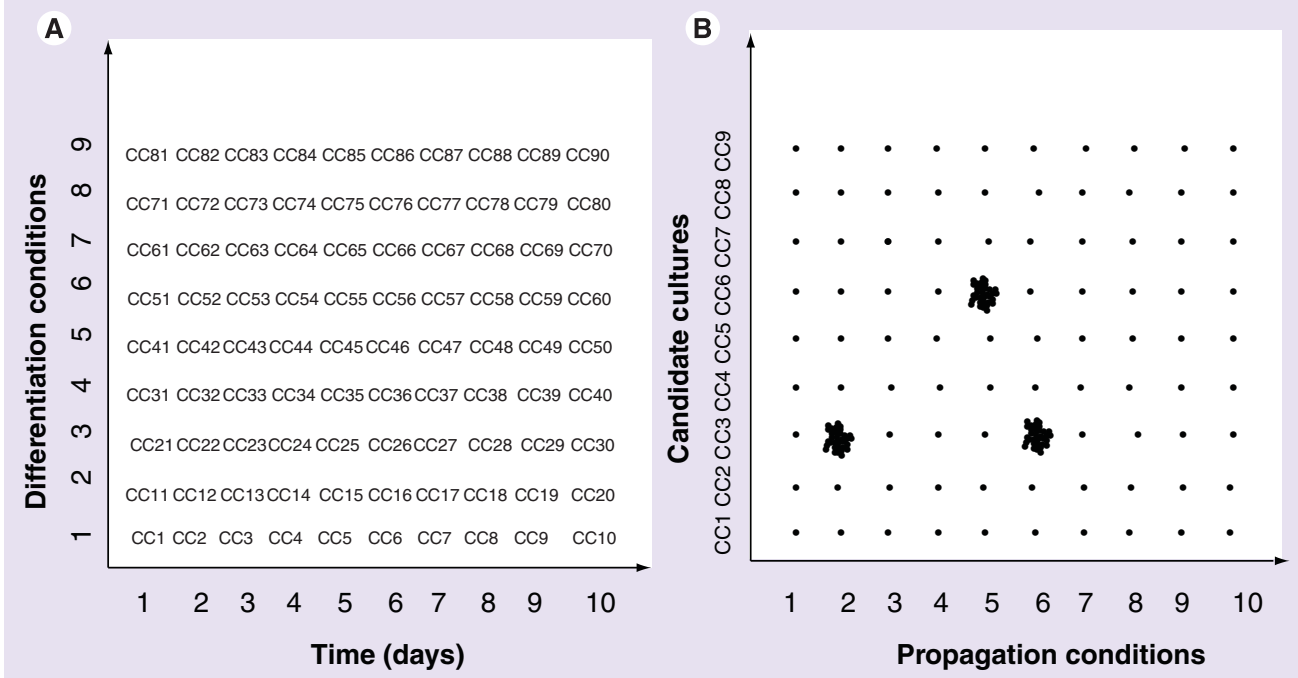

(A) In the first step, human embryonic stem cells are exposed to an array of differentiation conditions to generate diverse and heterogeneous subpopulations of embryonic progenitor cell types designated CCs. (B) In the second step, each CC subpopulation is plated at clonal densities in another array of media and growth factors to identify embryonic progenitor cell clones capable of long-term propagation.

CC: Candidate culture. 
Figure 2. Clonogenicity of human embryonic stem-embryonic progenitors derived by in situ colony differentiation.

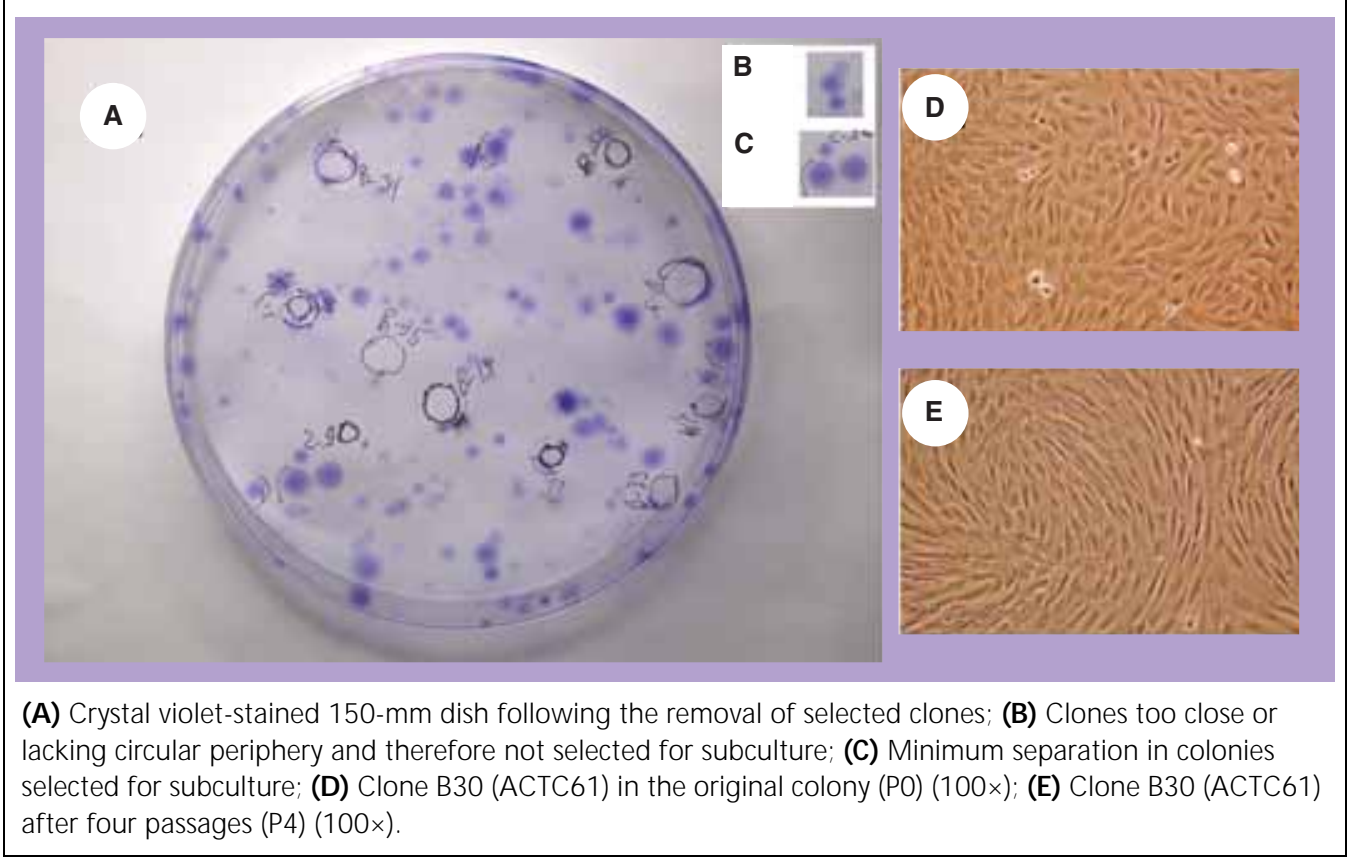

and often they can be expanded in culture and cryopreserved. H owever, their stability and uniformity over time were not studied. Each of these CCs were subsequently plated at clonal densities in an array of different cell culture media optimized for various stromal and epithelial cell types (Figre 1B). This two-step technique, when expanded to a large number of conditions, exposes $\mathrm{hES}$-derived cells to a very large number of combinations of conditions to capture cell lines without a previous understanding of the culture needs of any one of them. The final culture plates were left undisturbed for 14 days in $5 \%$ ambient oxygen and a total of 1090 robust colonies resulting from the combinations of conditions that appeared single cell-derived were removed with cloning cylinders and expanded (Figure2). The conditions under which each cell line was derived are summarized in supplementary TableAl Cells that did not display a uniform circular morphology or were too closely approximated to neighboring colonies were not selected for propagation (Figure2B), and visibly-distinct colonies were required for selection with a minimum separation similar to that of Figre2C. As can be seen in Figure2D \& E, the original colonies frequently showed highly mitotic and uniform populations of cells. A total of 280 lines $(25.7 \%$ ) expanded to at least four roller bottles and of these, approximately $80 \%$ cryopreserved/thawed well (as judged by the ability of thawed cells to be subsequently expanded at a propagation rate similar to that before freezing). Such cells were considered cell lines and assigned ACTC numbers (supplementary TadleAz).

\section{Gene-expression analysis}

To reduce variations in gene expression due to cell cycle artifacts, and to capture an early geneexpression profile of the cells, upon being expanded to six-well plates, cells were placed in media with a tenfold reduction in serum or similar growth supplements for 5 days and all were refed 2 days prior to harvest to reduce feeding artifacts. CD N A from each cell line was hybridized to microarrays for geneexpression analysis. CD N A from 242 cell lines (including three biological replicates for C4ELSR2, two biological replicates for the parental hES cell line H 9, two technical replicates of $X 2.2$ and two technical replicates of Z11) give a total of $242+9=251$ arrays.

CDNA was hybridized to either Illumina microbead arrays $\mathrm{H} 6 \mathrm{~V} 1$ and $\mathrm{H} 8 \mathrm{~V} 1$ (Illumina 1 ), Illumina H 6V 2 (Illumina 2) or Affymetrix U 133 Plus 2.0 (Affymetrix) and quantile normalized relative fluorescence units (RFUs) are shown in supplementary Tables A2, A3 \& A4. Included in the Illumina 1 data are results using the following controls from fully differentiated cell types: total brain RN A, human foreskin fibroblasts (Xgene) at passage 1 and 5 , purified CD $34^{+}$and CD $133^{+}$ peripheral blood lymphocytes and H 9 ES cell 
RN A. Average background signal was 140 RFU and 84 on the Illumina 1 and 2 platforms, respectively, and 9 RFUs on the Affymetrix arrays. The signal was considered positive if more than 200 RFUs on the Illumina 1 and 2 platforms and more than 100 on the Affymetrix arrays were shown (based on none of the background control probes showing RFU values greater or equal to these numbers). Since only 49 samples were analyzed by Affymetrix arrays, and such data could not be normalized to the Illumina samples, the Affymetrix data are shown in supplementary TadeA4 and generally not discussed in this report. The large number of cell lines made replicate microarray analysis economically unfeasible, therefore select microarray gene-expression levels were compared with that obtained by quantitative PCR (qPCR), demonstrating the probable reliability of the data (supplementary FigreAz). In addition, select cell lines were routinely repeated as technical replicates wherein the original RN A isolate was subjected to repeat microarray analysis, and biological replicates where the cell line was thawed, grown, RN A isolated and microarray analysis repeated, often by differing microarray core facilities and on different chips. Representative replicates included in this report are biological replicates repeated on the same chips of the parental hES cell line H 9 (WA09) (H 9 Biol and H 9 Bio2), three biological replicates of the hEP cell lines C4ELSR2 (Bio 1-3), two technical replicates of $\mathrm{X} 2.2$, two biological replicates of RAPEND 17 (Bio 1 being performed on Illumina 1 and Bio 2 on Affymetrix) and other technical replicates of the hEP cell lines 2-2 (Rep 1-2), Z11 (Rep 1-2), RASKEL18 (Rep 1 being performed on Illumina 1 and Rep 2 performed on Affymetrix) and W 8 (Rep 1 being performed on Illumina 1 and Rep 2 on Affymetrix; see supplementary Tables A1, A2, A3 \& A4). O ther biological and technical replicates were performed as a quality control, showing similar evidence of reproducibility (data not shown).

O btaining gene-expression data on so many clonal hES-derived cell lines allowed an unusual opportunity to determine what genes best control for constitutive expression in both hES cells and their differentiated progeny. 0 ften such data are normalized to the expression of a housekeeping gene such as GAPD; however, GAPD was never tested against in the context of large arrays and in the breadth of cell types derived in vitro from hES cells. We therefore sorted for genes

\section{Figure 3. Genes with relatively constitutive expression in diverse human embryonic stem-derived cells.}

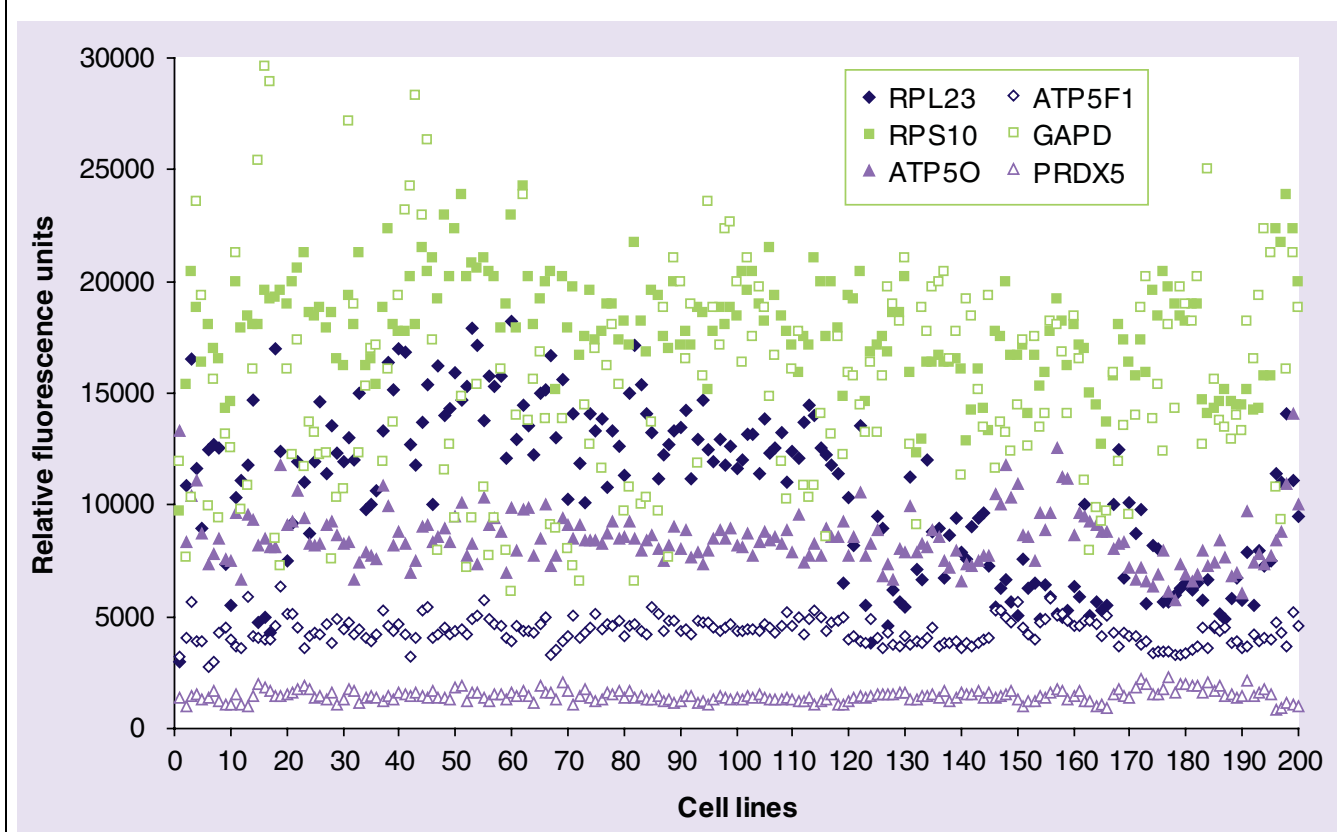

The relative expression of the genes RPL23, RPS10, ATP5O, ATP5F1 and PRDX5 from the Illumina 1 data set [supplementary Table A1] displayed less variability among the isolated human embryonic progenitor cell lines and human embryonic stem cells compared with the expression of the commonly used constitutive marker glyceraldehyde-3-phosphate dehydrogenase. 
with the lowest variation/RFU ratios (quantified as the standard deviation [SD] of RFU values/mean RFU values) and identified five candidate genes from the Illumina 1 data that displayed better constitutive expression when compared with GAPD (Figure3). It can be seen that while GAPD showed a SD/RFU value of 0.32, the ribosomal component genes RPL23 (SD/RFU of 0.12 ) and RPS10 (SD/RFU of 0.12 ), the ATP synthase subunits ATP50 (SD/RFU of 0.14 ) and ATP5F1 (SD/RFU of 0.13 ), and the antioxidant enzyme PRDX5 (SD/RFU of 0.14 ) were all better constitutive markers for hEP cell lines.

Clonal hEP cells do not display hES markers but instead show markers of diverse primitive embryonic progenitors To determine the nature and diversity of gene expression in the cultured hEP cell lines, genes in supplementary Tables A2, A3 \& A4 are rank ordered with genes with the largest RFU value/mean RFU value in all the hEP clones being at the top and the horizontal order of the cell lines reflects a hierarchical cluster order (i.e., cells with a similar pattern of gene expression are clustered together). $M$ arkers that are relatively highly expressed in each cell line compared with the other lines were determined by rank ordering the ratios of RFU values for each gene in that cell line/average RFU value of that gene for all cell lines (supplementary TableA5).

The Illumina 1 and 2 datasets were merged and hierarchically clustered based on sequences the two arrays had in common. Consistent with the cell lines appearing to be at least partially differentiated (i.e., not morphologically similar to the compacted colonies of hES cell lines), as shown in Figre4, the EP lines appeared to lack markers of hES cells such as OCT 4, although some of the lines expressed markers often associated with stem cells, such as CD 133 and CD 24. In addition, the majority of hEP cell clones expressed markers well known in mouse embryology to be important regulators of cell fate and expressed mainly in embryonic progenitors as opposed to fully differentiated tissues. For example, hierarchical clusters of cell lines expressed relatively high levels of MEOX1 and MEOX2, which are reported to be expressed in early embryonic mesoderm and neural crest derivatives [13,14]. The winged helix family of homeobox-containing factors are important in cell fate determination, pattern formation and organogenesis. Similarly, the winged helix factors, such as FOXF1 homologues, which are mainly expressed in a subset of developing fetal mesodermal cells in the mouse [15], are also expressed in various subsets of the hEP cell clones. A total of 136 of $192(71 \%)$ expression results in Illumina 1 data (supplementary TadleA2) showed RFU values of more than 200 (positive expression) for one of the three embryonic progenitor markers MEOX1, MEOX2 or FOXF 1, whereas none of the adult-derived brain, dermal fibroblast, lymphocyte or hES cell line samples studied expressed the genes. Additional embryonic markers, such as the winged helix factor FOXC1 that is reported to be expressed in cranial neural crest, paraxial mesoderm and somitomeres in the mouse but not adult tissues [16], was also highly expressed in numerous hEP cell clones. The gene for EN C1, which is mostly expressed in mouse neuroectodermal fated epiblast and brain, and to a lesser extent in some embryonic tissues such as brain, kidney, lung, heart and liver but exhibits diminished expression in the adult mammal $[17,18]$, is similarly expressed in a subset of the clones. 0 ther examples of embryo-specific genes expressed in the lines can be seen in supplementary Tables A2 , A3, A4\& A5, including the relatively high expression of $L H X 8$ in the cell line X7PEN D 16 (ACTC273) that is reported to be expressed only in the medial ganglionic eminence and perioral mesenchyme of the mouse in the middle embryonic to early postnatal development [19]. Further examples include ROR2, which is expressed in the mouse embryo but downregulated in the adult [20], SH OX2, which is expressed in embryonic CNS, cranial-facial mesenchyme, heart and limb mesoderm [21], and GPC2, an integral membrane HSPG expressed in immature neurons and downregulated subsequent to axon formation and terminal differentiation [22], as well as other embryo-specific genes. Evidence of the potential pluripotency of the clones is seen in the presence of markers of numerous differentiated cell types in some of the lines, such as the expression of GFAP, OLIG2 and neuronal markers (E68 [ACTC 207]).

The combined data from Illumina 1 and 2 were subjected to hierarchical clustering and the resulting dendrogram and an abbreviated heat map are shown in Figre5 (see supplementary FigreAz for a complete heat map, which includes a dendrogram correlating genes rather than cells). As seen in Figure5, genes that are expressed in relatively high levels are coded red and low levels of expression are blue. It can be seen that biological replicates of the human ES cell line $\mathrm{H} 9$ 
RESEARCH ARTICLE - West, Sargent, Long et al.

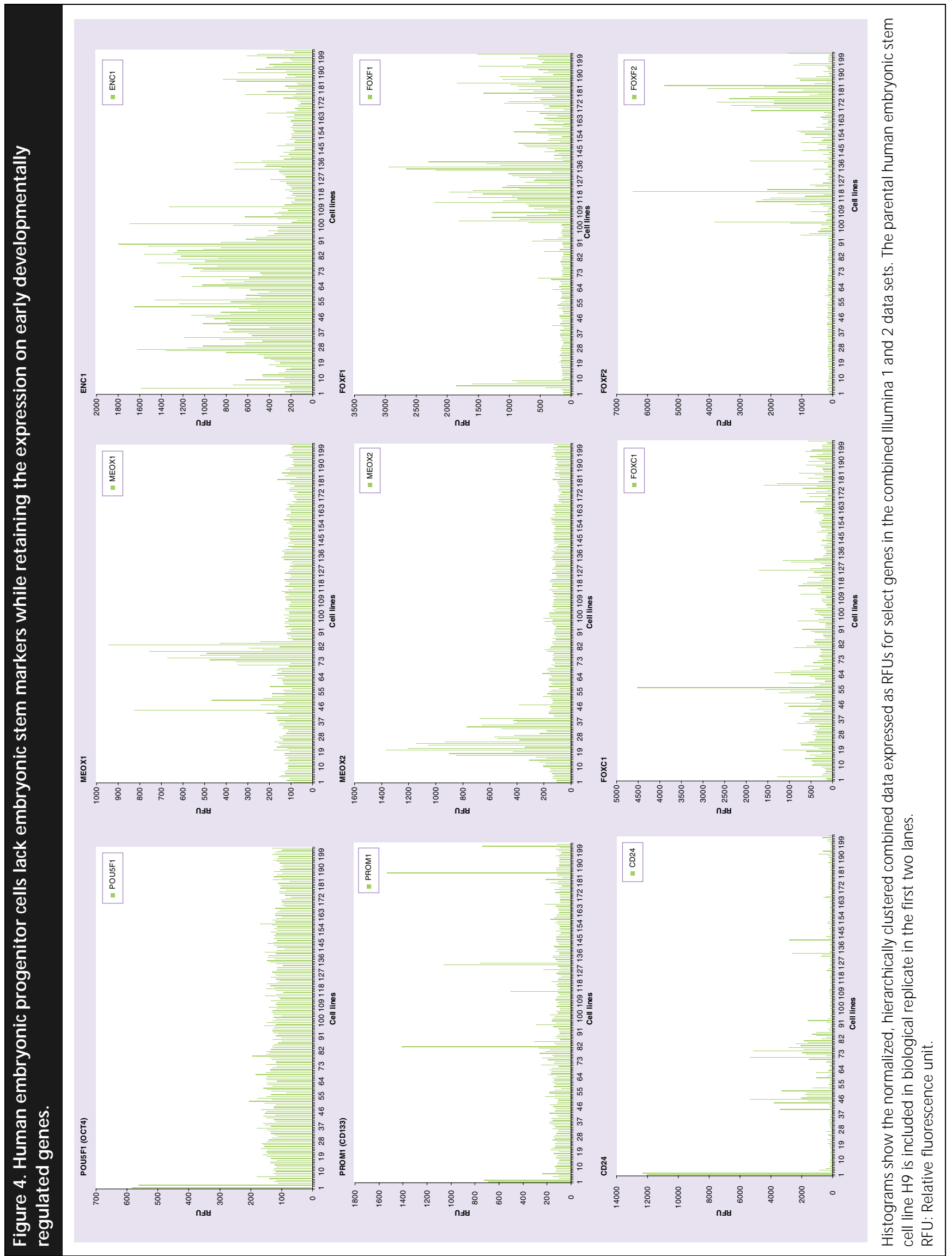


(WA09) clustered together and showed relatively high levels of CYP26A1, a P450 retinoic acidinactivating enzyme that while reported to play an important role in anterior-posterior positioning in the gastrulating embryo, has not been reported to be expressed at such high levels in cultured ES cells relative to their differentiated progeny [23]. The ES cells, but not the differentiated cell clones, also expressed relatively high levels of EBAF (lefty2 in the mouse), an inhibitor of nodal and reported to be rapidly downregulated following hES cell differentiation [24], as well as the transcription factors ZNF206 and ZIC3, both reported to be expressed at relatively high levels in hES cells but downregulated during differentiation and to play a role in maintaining an undifferentiated state $[25,26]$. It can be seen in Figure5 and supplementary FigureA3 that there are similar patterns of gene expression in the other biological and technical replicates but a wide array of different differentiated markers among the hEP cell lines. Examples include the genes PLP1, PM P2, GRIN 1 and GABRA1, typically expressed in neuroglial cells and highly expressed in the line E68 (ACTC207). Other examples are the gene Myosin $\mathrm{Va}$, which is involved in the transport of secretory vesicles of neurons and melanocytes [27], GARP, which is expressed at relatively high levels during murine embryogenesis such as in limb dermis, smooth muscle and vascular endothelial cells [28], EDIL3, which is reported to be involved in the embryonic regulation of vascular morphogenesis [29], Col24A1, which is relatively specific to developing bone and cornea [30], and SEM A5A, which is expressed by oligodendrocytes [31]. Other selected markers for other lines are shown in Figure 5 and supplementary FigreA3. The expression of these markers, while not definitively diagnostic of the cell types discussed, nevertheless provides evidence of the diversity of cell types that can be propagated clonally from hES cell lines in vitro.

The diversity of clonal derivatives can also be seen through the specific expression of homeobox genes. All differentiated cells, for example dermal fibroblasts [11], have the potential to vary widely in gene expression from one geographic location in the body to another depending on DLX, MEOX, HOX, LIM, MSX, BAPX, PRRX, GSC, IRX, SOX, PIT X and FOX gene expression. As can be seen in Figre6, there is a diversity of homeobox gene expression in the hEP cell lines, perhaps reflecting the fact that while there are multiple isolates of lateral plate mesoderm, for example, differences in $\mathrm{H}$ OX gene expression are resulting in subtle differences in extracellular matrix and other proteins that lead to the cells being grouped as unique cell types.

To provide an objective measure of the complexity of the hEP cell library, a grouping using non-negative matrix factorization (N M F) analysis was performed. Thek-value was incrementally altered to obtain the highest stability score without scattering known biological replicates (three independent isolations of ELSR2, two biological replicates of $\mathrm{H} 9$ and two technical replicates of Z11). The stability scores where k-values range from 100 to 145 are shown in supplementary FigreA2 and the resulting N M F plot is shown in Figure7. The cells were assigned group numbers and these group numbers, as well as the order in which the cells are displayed in the N M F plot, are shown in supplementary TableAl The most stable k-value was 140 ( $k=142$ less two samples excluded from this report), suggesting that the complexity of the cell lines analyzed on the Illumina platform was 140 . Consistent with this conclusion, the cells within a given group have similar marker genes and cluster together (Figre5). For example, the cells of group 30 (E84, $E 30, E 3, E 73, E 57$ and E67) all have a similar pattern of geneexpression markers such as S100A4 (supplementary TableA5) and cluster as a discrete group by hierarchical clustering (Figure5). Also, the N M F analysis did not split biological or technical replicates. The cell lines analyzed with Affymetrix arrays could not be combined with those lines analyzed with Illumina arrays in the N M F analysis, therefore the estimated complexity is restricted to those cell lines assayed on the Illumina platform. H owever, because at least one line (MEL2 [ACTC 268]) analyzed on the Affymetrix arrays displays numerous unique markers not seen in any cell line analyzed on Illumina bead arrays, but it appears to include cell lines with markers not characterized on the Illumina platform, we conclude that the number of distinguishable hEP cell cultures isolated and described in supplementary TableAl were more than 140.

Immunocytochemical confirmation of hEP microarray gene-expression analysis The microarray gene-expression data suggested that the hEP cell lines express profiles of numerous primitive neural crest, endodermal, mesodermal or ectodermal lineages. To determine whether protein expression of several unique markers of differentiation correlated with the relatively high 


\section{Figure 5. Abbreviated heat map of common gene sequences on Illumina 1 and 2 platforms for hierarchically} clustered cell lines.

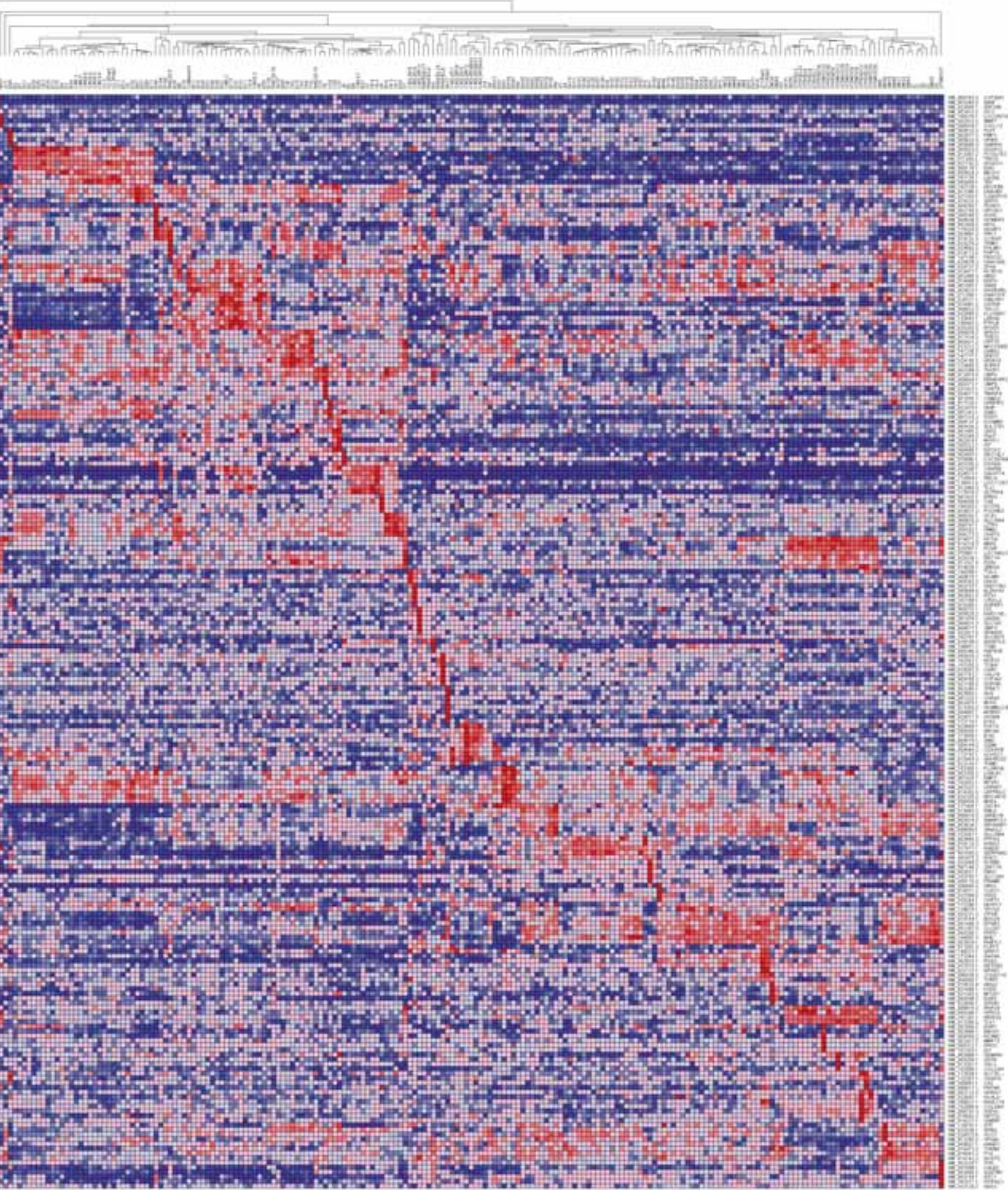

Human embryonic stem (hES) cells and derived human embryonic progenitor cell clones, normalized and hierarchically clustered, with the resulting dendrogram and heat map of select genes. Relatively highly expressed genes are shown in red and genes not expressed are blue. The parental hES cell line $\mathrm{H} 9$ is included in biological replicate in the first two columns. 


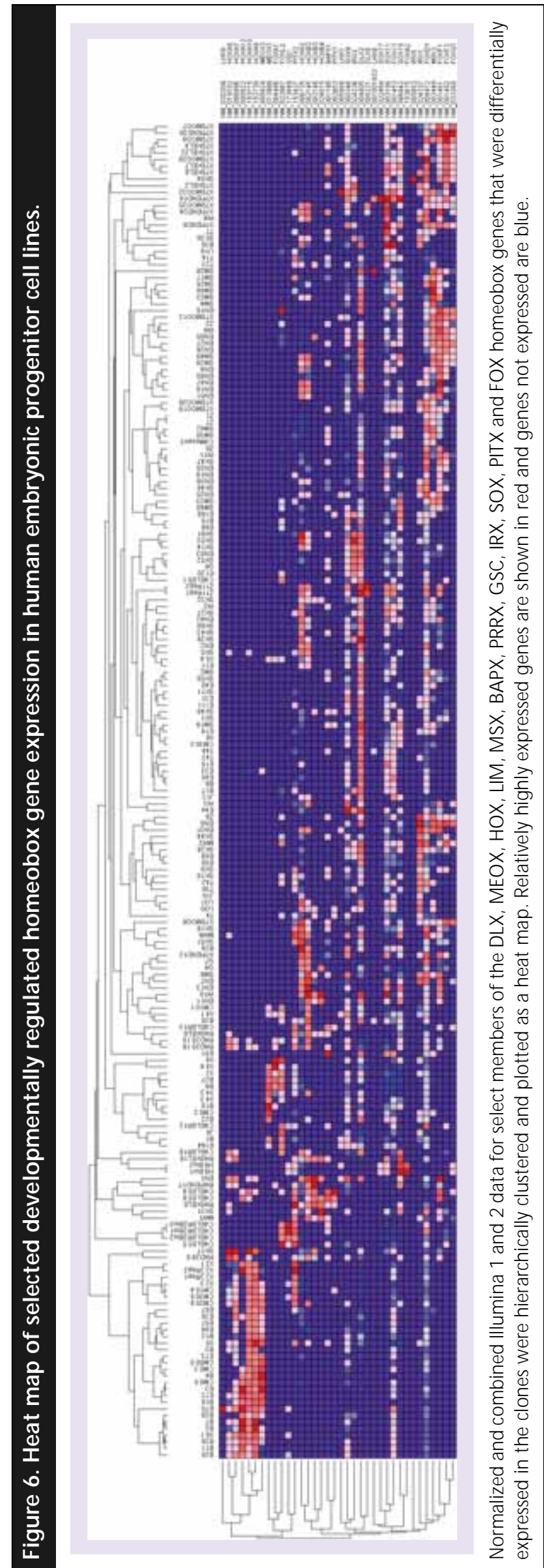

RNA expression levels of the markers in hEP cell lines, we used immunocytochemical analysis. In each of the four hEP cell lines shown, proteins corresponding to highly expressed mRN As were readily detected by immunocytochemical staining with the appropriate antibody (Figures8 $\&$ 9). Accordingly, the cell line 7PEN D 24

(ACTC 283) expressed genes consistent with being a neural crest line, such as the melanocyte markers TYRP1 and EDNRB, peripheral neuron markers such as EGR2, STMN2, DCX, CNTNAP2, GPC2 and PROM 1, and cartilage markers, such as CILP (see supplementary Tade A5). The neural progenitor markers NES [32] and CNTN 6 [33] were confirmed on a protein level with specific antibodies in the cell line corresponding with mRN A expression (Figre8A-F). A typical intermediate filament staining pattern for NES was observed under high power (Figre8B). In the case of the cell line 7PEND24, the most caudal HOX gene expression was HOXA2 and HOXB2, suggesting it corresponded to an origin in the hindbrain.

The cell line $M 10$ (ACTC103) expressed relatively high levels of FOXA2, TCF2(HNF1B) and NMES1 (supplementary TadleA5), consistent with the cells being endodermal, possibly oral or esophageal epithelia in nature [34-36]. The genes $\alpha$-fetoprotein and keratin 20 [37] were also expressed at relatively high levels and the corresponding proteins were confirmed to also be expressed using specific antibodies (Figre8G-L). A typical keratin filament staining pattern was observed under high power (Figresk). The most caudal HOX gene expression was HOXB5, suggesting that the cell line is foregut in nature.

The mesodermal marker MYH 3 and intermediate filament NES, both of which are known to be expressed in embryonic but not adult heart and skeletal muscle [38,39], were detected in the SK 17 (ACTC162) cell line, which expressed both proteins at detectable levels (Figre 9A-F). The M YH 3 staining of SK 17 resulted in a staining pattern with myocytelike microfilament morphology (Figure9A\& B). The cells also expressed relatively high levels of ACTC, MYBPH, TNNC1, MYOD 1 and HUMMLC2B (supplementary TadeA6) and the most caudal HOX gene expression was HOXA11, HOXB9 and HOXC6. Only the large cells stained positive for MYH3, suggestive of a more primitive cell type in the cultures as well. Interestingly, SK 17 also expressed cardiac M YH 7 and markers normally associated with cardiac cells such as CASQ 2, TN N T 2, neuronal cell types (e.g., NEF3) and axon guidance molecules (e.g., SPON 1, SLIT2 and RT N 4; supplementary TableA5). The expression of these neuronal markers and the unique and strong expression of M YBPH, which is expressed in skeletal and heart conduction fibers, and SLN, which is expressed in soleus and artial but not ventricular cardiac muscle, suggests that these cells may be a previously unrecognized cardiac progenitor, perhaps playing a role in the conduction system of the heart.

As previously discussed, the cell line E68 (ACTC207) expressed numerous geneexpression markers of neuroglial 
Figure 7. Non-negative matrix factorization plot of cell clones analyzed on the Illumina platform.

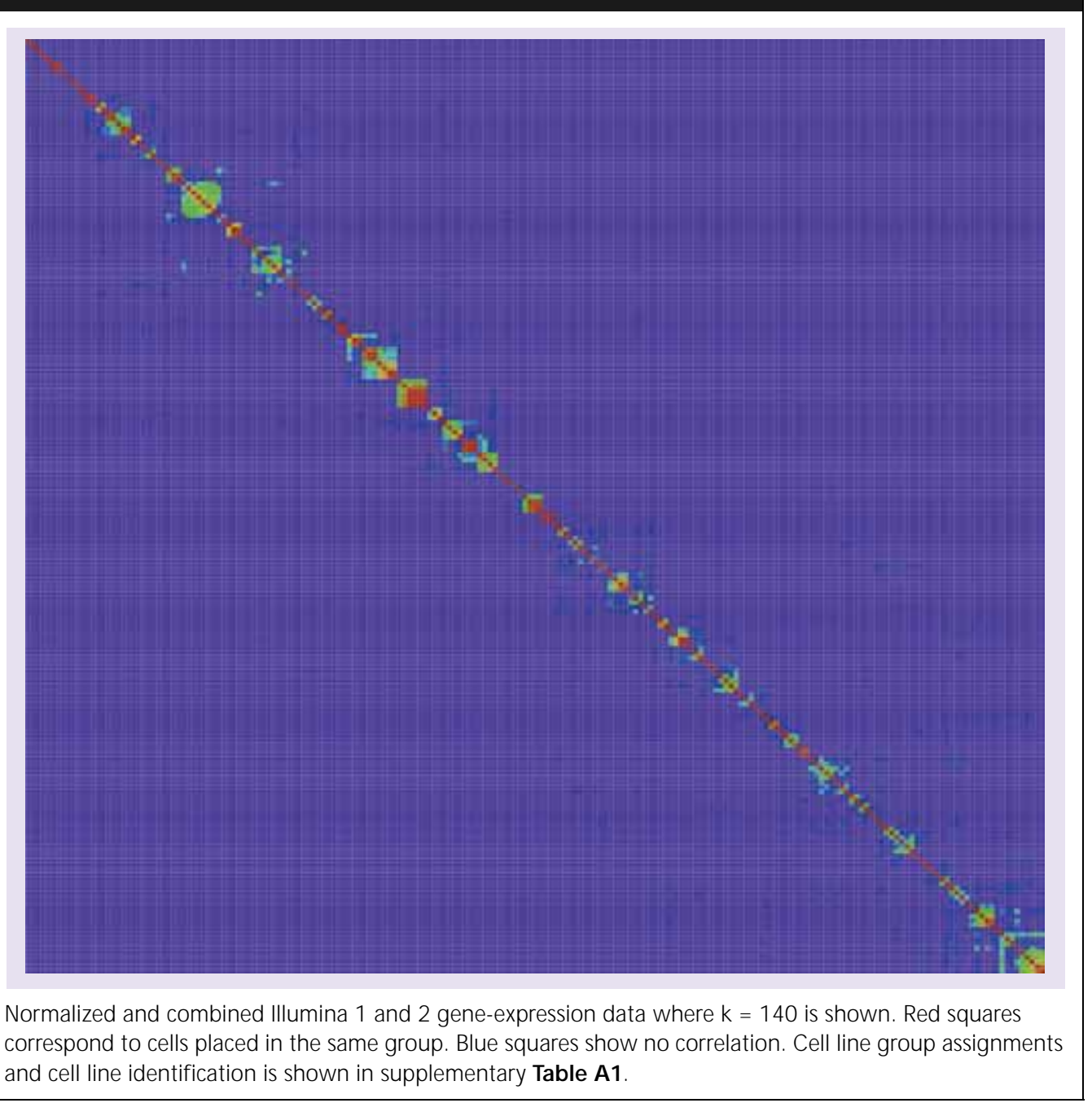

lineages but lacked HOX gene expression. The ectodermal markers synaptosomal-associated protein 25 and CTNTN 6 were detected on a protein level in the E68 cell line that expressed high levels of both marker mRN As (Figure9G-L). For the detection of each of the previously discussed marker proteins, substitution of the primary antibody with an isotype-matched control antibody resulted in little or no detection of fluorescent secondary antibody binding (Figures $8 \mathbf{C}, \mathbf{F}$, $\mathbf{I}, \mathbf{L} \& \mathbf{9 C}, \mathbf{F}, \mathbf{I}, \mathbf{L})$. O verall, protein markers of differentiation were appropriately expressed in those $h E P$ lines that overexpressed the corresponding marker gene.

The transfer of E68 to neurobasal medium supplemented with $\mathrm{N} 2$ for 57 days altered the proliferative population of stellate cells (Figure10A) to cells with a more neuroglial morphology, including clusters of mutually adherent cells resembling neurospheres (Figure10B), cells displaying growth conelike structures (Figre 10C) and cells with structures resembling synapses (Figure10D), consistent with the immunocytochemical markers shown for $E 68$ in Figre9G-L and the gene-expression markers observed in the line (supplementary TableA5), although further physiological studies of the cells to confirm neuron-like activity is warranted.

Clonal hEP lines express diverse cell surface antigens

The use of affinity methods to purify cell lineages has often been used in hematology. We therefore investigated whether hEP cell lines that showed differentially expressed CD antigens predicted the presence of these antigens on the cell surface, potentially facilitating the repeated isolation of desired clones. As seen in 
The ACTC ellerate initiative - RESEARCH ARTICLE

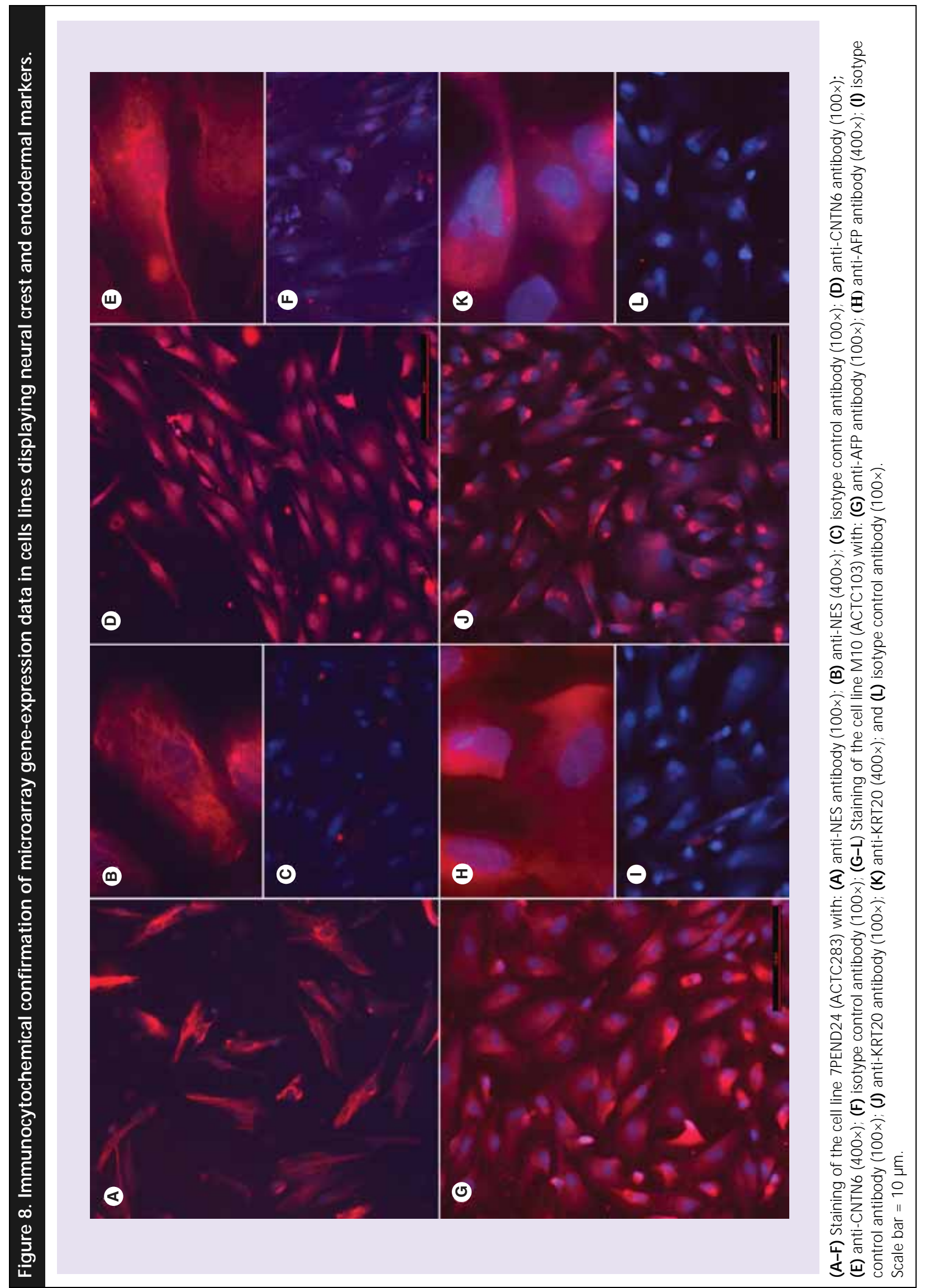


supplementary Table A6, CD antigen gene expression varied widely among the cell lines. We then compared the percent positive cells as determined by flow cytometry with the expression of selected CD antigens in a subset of the cell lines. By gene expression, CD 81 was strongly expressed in all the lines and as seen in Table1, all cell lines were positive for this antigen. By contrast, CD 24 gene expression in 4D 20.8 (ACTC 84) was weakly positive, E68 (ACTC207) was strongly positive, E109 (ACTC 117) was negative, ELS5.8 (ACTC 238) was negative, ELSR 10 (ACTC 152) was negative, M 10 (ACTC 103) was negative, 7PEND 24 (ACTC283) was negative and SK 17 (ACTC 162) was positive. As seen in Tablel, $30.4 \%$ of $4 D 20.8,94.2 \%$ of $E 68$ and $45.6 \%$ of $\mathrm{M} 10$ cells were positive, but the other lines were negative. Interestingly, the CD 24 antigen distinguished the hindbrain neural crest neural progenitor line 7PEN D 24 (CD 24-) from the H OXneural progenitor line E68 $\left(C D 24^{+}\right)$, demonstrating the usefulness of clonally isolated hEP lines in potentially identifying useful cell surface antigens. The variability of expression of $C D$ antigens in differentiated hEP cell lines may be a result of continued differentiation of the cells subsequent to clonal isolation and underscores the need for additional study.

\section{hEP clones express unique secreted factors}

Embryonic cells express a host of secreted factors that regulate complex organogenesis. We profiled those genes known to be processed as secreted proteins and those genes differentially expressed in each line are summarized in supplementary TableA7. It can be seen that the isolated hEP cell clones show expression of a wide array of transcripts for growth factors, cytokines, proteases, protease inhibitors and extracellular matrix factors. We then selected an arbitrary subset of the lines and performed ELISA to determine whether we could confirm protein expression in the conditioned medium. Geneexpression profile data suggest that the cell lines EN 13 and 47 are expressing AREG in relatively high levels, whereas the cell lines SK 17 and Xgene fibroblasts express very little or no AREG. This observation is validated on a protein level as seen in supplementary Table A8, where the lines EN 13 and 47 expressed 6.35 and $6.36 \mathrm{ng} / \mathrm{ml}$, respectively, in $72 \mathrm{~h}$ conditioned medium and SK 17 and Xgene were negative. Similarly, geneexpression profile data also suggest that the cell line ELSR 10 may be secreting the following factors: FGF-7, IGFBP-5, PDGF-BB, TGF- $\beta 1$, TIM P-1 and vitronectin. Since some of the factors may be secreted in small amounts, below the detection level, the cell culture medium was concentrated fivefold using a Millipore U Itrafree concentrator (Thermo Fisher Cat. No. UFV 5 $B C C$ 25) with a $5000 \mathrm{MW}$ cutoff. Medium from the cell lines EN 13, EN 47, SK 17 and $X$ gene fibroblasts were tested simultaneously for the same factors. Results shown in supplementary TableA8also validate the gene-expression levels in that the cell line ELSR 10 alone expressed high levels of all of these factors relative to the nonexpressing cell lines.

\section{hEP cells lack tumorigenicity}

W hile hES cells generate benign teratomas when injected into immunocompromised animals, the tumorigenicity of purified hEP types has not been extensively studied. The examination of genes expressed at relatively high levels in each line revealed numerous genes known primarily for their expression in malignancies and in embryonic development (oncofetal genes). For example, SILV is reportedly expressed in a large number of melanomas [40] and in embryonic retinal pigment epithelium and neural crest-derived melanoblasts [41] and is expressed at relatively high levels in SK 17 (ACTC162). Other such oncofetal genes expressed in the isolated hEP cell lines include PLAG1, AMIG02, HCLS1, SPINK1，PRAME， INSM 1，RAGE， ENC1, BCAS1, GRM 1, TSGA10, S100A2, A4, A6, GPC3，EGFL6，PSG 5, CEACAM 1, CGPC3, SRPUL, DCDC2, LRRN 5, SOX11, RUNX3, CA12, STARD 10, CXCL1, ANPEP, GAGE6, NCOA6, TACSTD 2 and TSPAN 8. We therefore tested the tumorigenicity of an arbitrary group of the hEP cell lines in severe combined immunodeficiency (SCID) mice. A total of 20 million cells from each of the cell lines B16 (ACTC 59), B28 (ACTC60), 6-1 (ACTC64), B26 (ACTC50), B11 (АCTC58), B2 (АCTC51), CM 02 (АCTC 77), E75 (АCTC 102), E15 (ACTC 98), 4D20.9 (ACTC 82), E72 (ACTC 100), EN 7 (АCTC 184), EN 55 (ACTC185), SK 17 (ACTC 162) and Z11 (ACTC194) were injected (each cell line injected into two SCID mice with approximately 10 million cells/mouse or a total of 30 mice and 60 injection sites). H alf the cells (5 million) were injected intramuscularly into the right rear leg and the other 5 million subcutaneously into the left rear leg. After 
The ACTC ellerate initiative - RESEARCH ARTICLE

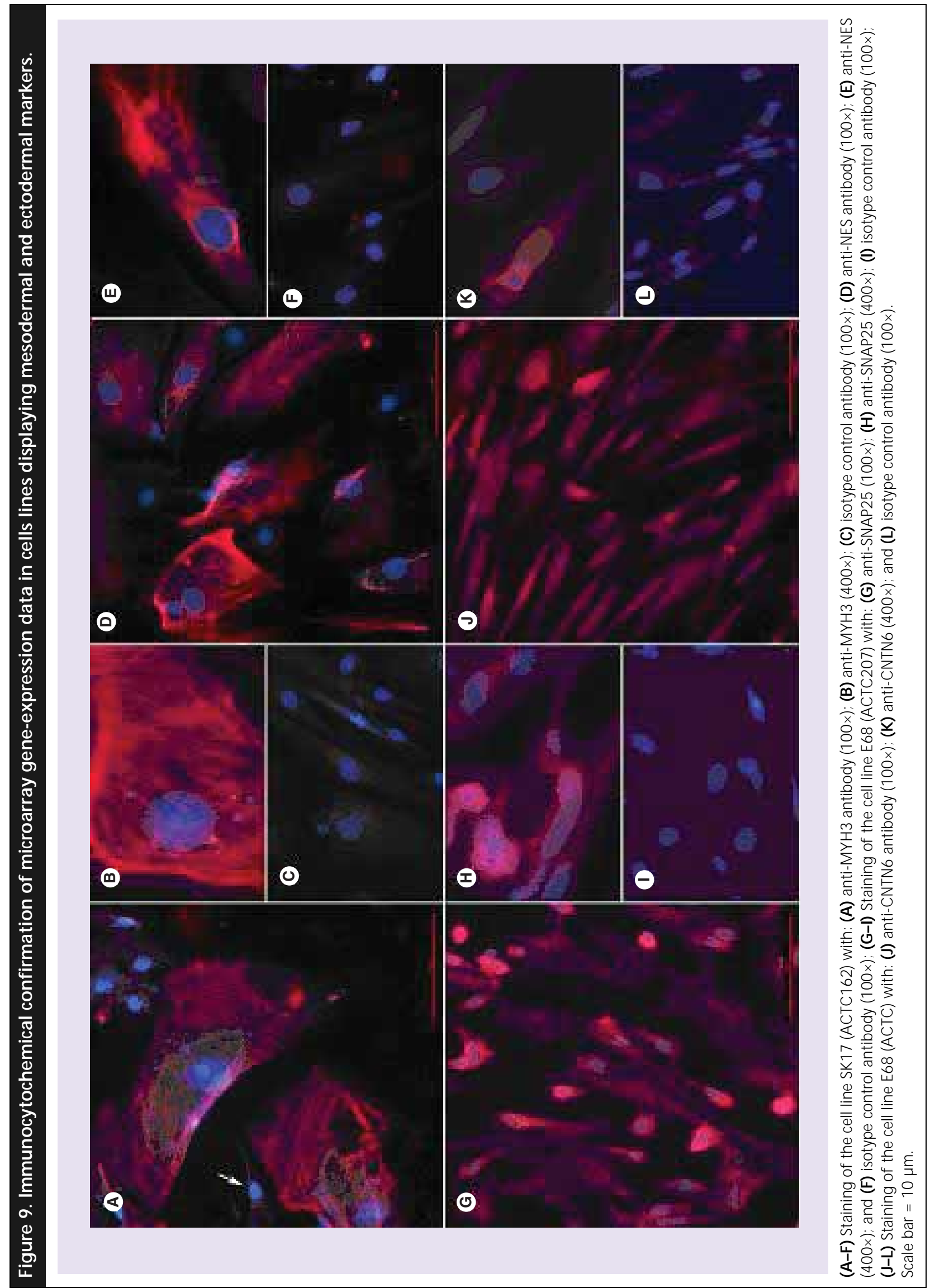


4-6 months, a thorough pathological analysis could reveal no grossly visible abnormalities, dehydration, malnutrition, lesions, hair loss, inflammation or any other evidence of past or current disease process and upon dissection, there was no evidence of tumors, congregation, redness necrosis or edema in the limbs, abdomen, thoracic cavity or neck. O ne exception was the cell line B28, which showed an approximately $1 \mathrm{~mm}$ nodule between the skin and leg muscle near the site of injection. In our experience, the injection of similar numbers of hES cells at these sites and for these periods of time would have led to teratoma formation in the majority of animals.

hEP cells include clones with a robust \& mortal proliferative capacity H uman germline cells, such as sperm, show relatively long and stable mean telomere terminal restriction fragment (TRF) lengths of $12-15 \mathrm{kbp}$ [42]. H uman ES cells are likely unique among cultured normal human cells in maintaining germline telomere length through the activity of telomerase [1]. We therefore assayed selected early hES-derived hEP cell clones for telomere length by Southern analysis and telomerase activity by the telomeric repeat amplification protocol (TRAP) assay during extended passaging in vitro to provide insight into the proliferation potential of the lines compared with normal human cells of neonatal origin. As shown in Figrewa, the lines EN 13, SK 17, SM 28 and SM 22 were propagated and compared with a neonatal foreskin fibroblast cell line X gene. With the exception of the line SK 17, all clonal hEP cell lines showed equal or greater proliferative capacity than the nonclonal neonatal foreskin fibroblasts. Since the majority of human cell clones generally senesce 20 or more doublings earlier than the mass culture from which they were derived (i.e., mass cultures proliferate to the limit of the longest-lived constituent clone), and most human cell clones isolated from neonatal or adult sources senesce in less than 50 population doubling (PD), we conclude that hEP cell clones studied herein may markedly exceed the proliferative capacity of cells derived from neonatal or adult sources. As shown in Figre 11B, a Southern blot of telomere lengths of the parental hES cell line $\mathrm{H} 9$ versus hEP cell clones isolated from that line shows that telomere length is germline in length in the line $\mathrm{H} 9$ and subsequently shortens in all hEP cell clones studied. As shown in Figurenc, the initial telomere lengths appear to be higher in the cell clones at early passage compared with early passage fore skin fibroblasts (Xgene) despite being clonally isolated, and the mean rate of loss was comparable in the lines with the exception of SK 17, which showed an accelerated loss, likely due to poor plating efficiency and/or apoptosis (data not shown). Telomerase activity was high in the hES cell line $\mathrm{H}$ 9, but low or negative in all hEP cell lines at all passages measured (supplementary FigureA4).

\section{Methods}

hES cell culture \& generation of

candidate cultures

The hES cell lines used in this study were previously described $\mathrm{H} 9$ (NIH registered as WA09) and the line (M A03) derived at Advanced Cell Technology (CA, USA). hES cells were routinely cultured in hES medium knockout Dulbecco's modified Eagle's medium (D M EM, Invitrogen, Carlsbad, CA, USA), IX nonessential amino acids (Invitrogen), 1X Glutamax-1 (Invitrogen), $55 \mu \mathrm{M} \quad \beta$-mercaptoethanol (Invitrogen), 8\% knockout serum replacement (Invitrogen), 8\% Plasmanate, $10 \mathrm{ng} / \mathrm{ml}$ LIF (M illipore, Billerica, MA, USA), $4 \mathrm{ng} / \mathrm{ml}$ basic FGF (Millipore), $50 \mathrm{unit} / \mathrm{ml}$ penicillin and 50 units $/ \mathrm{ml}$ streptomycin (Invitrogen). The cells lines are maintained in, and all subsequent experiments are carried out at, $37^{\circ} \mathrm{C}$ in an atmosphere of $10 \% \mathrm{CO}_{2}$ and $5 \%$ $\mathrm{O}_{2}$ on mitomycin $\mathrm{C}$-treated mouse embryonic fibroblasts (MEFs) and passaged by trypsinization. hES cells were plated at 500-10,000 cells per $15 \mathrm{~cm}$ dish. CC differentiation experiments were performed with either adherent hES cells grown on M EFs or with hES EBs. For adherent differentiation experiments, hES cells were allowed to grow to confluence and differentiated by a variety of methods described in supplementary TableAl For example, in the case of colony in situ differentiation in DMEM with $10 \%$ fetal calf serum, growth medium was replaced with DM EM containing $10 \%$ fetal bovine serum for differentiation and after various time periods ( 1 , $2,3,4,5,7$ and 9 days in differentiation medium) the cells are dissociated with $0.25 \%$ trypsin (Invitrogen) and plated in $150 \mathrm{~cm}^{2}$ flasks for expansion. The candidate cells from each time point in the $150 \mathrm{~cm}^{2}$ flasks were plated out for cloning and expansion as described below. For EB differentiation experiments, confluent hES cultures were treated for $15 \mathrm{~min}$ at $37^{\circ} \mathrm{C}$ with $1 \mathrm{mg} / \mathrm{ml}$ collagenase IV (in D M EM, Invitrogen) to release the colonies. The detached, intact colonies were scraped and collected by 


\section{Figure 10. Induction of neuronal differentiation.}
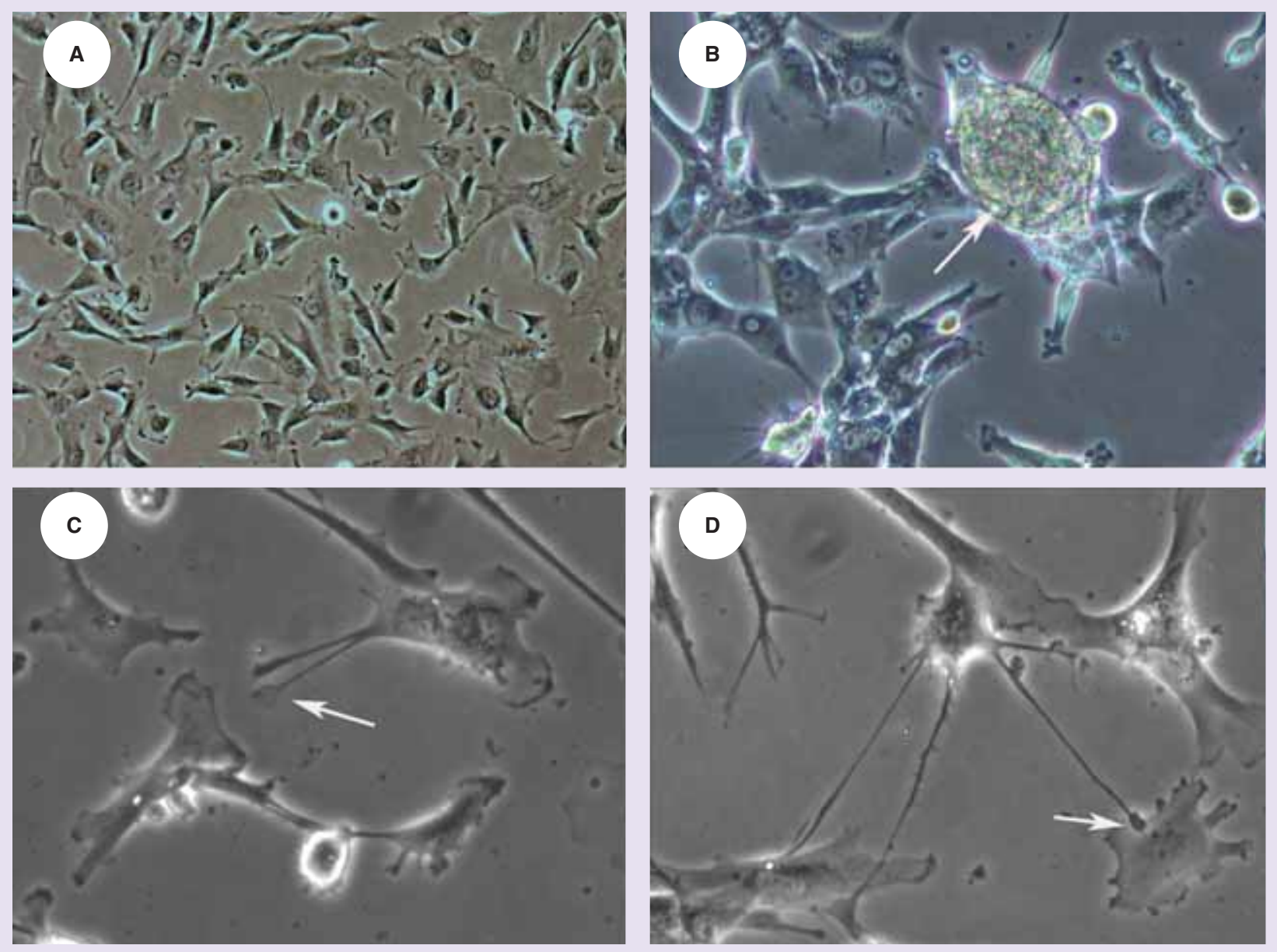

(A) Cell line E68 (ACTC207) at passage 19 in the derivation media (100x); (B) E68 at 57 days in neural induction medium (arrow: structures resembling compacted neuroepithelium) (200x); (C) E68 at 57 days in neural induction medium (arrow: structures resembling growth cones) (400x) and (D) E68 at 57 days in neural induction medium (arrow: synapse-like structures) (400X).

centrifugation (150 xg for $5 \mathrm{~min}$ ), resuspended in differentiation medium described in Table1 and transferred to a single well of a six-well ultra-low binding plate (Corning, distributed by Fisher Scientific, Pittsburgh, PA, USA) containing the same differentiation medium. The EBs were allowed to differentiate, depending on the experiment, from 4-7 days and the differentiated EBs dissociated with $0.25 \%$ trypsin and plated in sixwell plates containing various expansion medium. The CCs in the six-well plates are allowed to grow to confluence and plated out for cloning and expansion as described later.

Isolation \& expansion of clonal cell lines The differentiated candidate cell cultures described previously were dissociated with $0.25 \%$ trypsin to single cells and plated onto duplicate $15-\mathrm{cm}$ gelatin-coated plates at cloning densities of approximately 500 and/or 1000 and/or 2000 and/or 5000 cells per plate for further differentiation and expansion in a variety of growth media described in supplementary TableAl The clonal density cells were allowed to grow, undisturbed, for 10-14 days and colonies that developed were identified and collected with cloning cylinders and trypsin using standard techniques [10]. The cloned colonies were transferred onto gelatin-coated 24-well plates for expansion. As the clones become confluent in the 24-well plates, they were sequentially expanded to 12-well, six-well, T-25 flask, T-75 flask, $\mathrm{T}-150$ or T-225 flasks and, finally, roller bottles. Clonal cell lines that expand to the roller bottle stage are assigned a unique ACTC identification number, photographed and cryopreserved in 


\begin{tabular}{|c|c|c|c|c|c|c|c|c|}
\hline & $\begin{array}{l}\text { 4D20.8 } \\
\text { p11 }\end{array}$ & $\begin{array}{l}\text { E68 } \\
\text { p14 }\end{array}$ & $\begin{array}{l}\text { E109 } \\
\text { p10 }\end{array}$ & $\begin{array}{l}\text { ELS5.8 } \\
\text { p10 }\end{array}$ & $\begin{array}{l}\text { ELSR10 } \\
\text { p5 }\end{array}$ & $\begin{array}{l}\text { M10 } \\
\text { p8 }\end{array}$ & $\begin{array}{l}\text { 7PEND24 } \\
\text { p10 }\end{array}$ & $\begin{array}{l}\text { SK17 } \\
\text { p13 }\end{array}$ \\
\hline $\begin{array}{l}\text { CD24 } \\
(\mathrm{mlgG} 1)\end{array}$ & 30.4 & 94.2 & 0.0 & 1.1 & 5.7 & 45.6 & 0.0 & 0.0 \\
\hline $\begin{array}{l}\text { CD49b/ITGa2 } \\
(\mathrm{mlgG} 2 \mathrm{a})\end{array}$ & 49.7 & 12.6 & 19.7 & 95.6 & 92.3 & 56.0 & 97.1 & 82.8 \\
\hline $\begin{array}{l}\text { CD66a/CEACAM } 1 \\
(\mathrm{mlgG} 2 \mathrm{~b})\end{array}$ & 3.0 & 3.6 & 1.8 & 9.3 & 6.1 & 12.1 & 1.8 & 4.3 \\
\hline $\begin{array}{l}\text { CD81/TAPA-1 } \\
(\mathrm{mlgG} 1)\end{array}$ & 97.4 & 97.6 & 34.8 & 94.6 & 97.2 & 87.6 & 95.6 & 90.0 \\
\hline $\begin{array}{l}\text { CD104/ITGb4 } \\
\text { (mlgG } 1)\end{array}$ & 4.4 & 4.1 & 0.3 & 7.1 & 4.8 & 5.5 & 0.8 & 0.0 \\
\hline $\begin{array}{l}\text { CD117/kit } \\
(\mathrm{mlgG} 1)\end{array}$ & 0.0 & 4.4 & 0.0 & 0.0 & 0.0 & 0.0 & 0.0 & 0.0 \\
\hline $\begin{array}{l}\text { CD133/prominin } \\
\text { (mlgG1) }\end{array}$ & 4.0 & 3.5 & 4.9 & 7.0 & 5.7 & 1.3 & 0.6 & 1.5 \\
\hline $\begin{array}{l}\text { CD184/CXCR4 } \\
\text { (mlgG2a) }\end{array}$ & 2.5 & 1.5 & 2.1 & 0.0 & 2.8 & 6.9 & 1.2 & 1.0 \\
\hline $\begin{array}{l}\text { CD252/OX40L } \\
\text { (mlgG 1; mlgG1 IsoCon, mlgG2a } \\
\text { IsoCon, mlgG2b IsoCon) }\end{array}$ & $\begin{array}{l}5.4(2.2 \\
1.0,0.2)\end{array}$ & $\begin{array}{l}3.2(1.8 \\
0.4,0.4)\end{array}$ & $\begin{array}{l}3.1(4.1 \\
1.5,1.2)\end{array}$ & $\begin{array}{l}5.2(4.4 \\
1.8,0.1)\end{array}$ & $\begin{array}{l}4.1(1.6 \\
0.3,0.1)\end{array}$ & $\begin{array}{l}7.6(7.4 \\
3.4,1.3)\end{array}$ & $\begin{array}{l}0.0(3.9 \\
2.4,1.6)\end{array}$ & $\begin{array}{l}1.7(8.6 \\
2.4,1.3)\end{array}$ \\
\hline
\end{tabular}

\footnotetext{
*\% positive cells calculated by subtraction of percentage of isotype control Ig-labeled cells (shown) in gated region from specifically stained cells.
}

aliquots for later use. O nce cells reached a confluent $\mathrm{T}-25$ flask, they were passaged to a $\mathrm{T}-75$ flask and a fraction of the cells $\left(5 \times 10^{5}\right)$ were removed for plating in a gelatinized 6 - $\mathrm{cm}$ dish for gene-expression profile analysis. Following removal of the cell clones from the cloning plates, remaining colonies were visualized by crystal violet staining (Sigma HT9132-1L) in $100 \%$ ethanol per manufacturer's instructions. Cell culture media utilized in experiments and described in text and Table1: smooth muscle cell basal medium (Cat. N o. C-22062B) and growth supplement (Cat. N o. C-39267), skeletal muscle basal medium (Cat. No. C-22060B) and growth supplement (C at. No. C-39365), endothelial cell basal medium (Cat. $\mathrm{No}$. C -22221) and growth supplement (C at. N o. C-39221), melanocyte cell basal medium (C at. N o. C -24010B) and growth supplement (Cat. No. C -39415) were obtained from PromoC ell $\mathrm{GmbH}$ (H eidelberg, Germany). Epi-Life ${ }^{\mathrm{TM}}$, calcium free/phenol red-free medium ( $\mathrm{C}$ at. $\mathrm{No}$. $M-E P I C f / P R F-500)$ and low serum growth supplement (Cat. N o. S-003-10) were purchased from Cascade Biologics (Portland, OR, USA). M esencult basal medium (Cat. N 0. 05041) and supplement ( $\mathrm{C}$ at. No. 5402) were obtained from Stem Cell Technologies (Vancouver, BC,
USA). N eural induction medium was neurobasal medium (C at. N o. 12348-017) with N 2 purchased from Invitrogen. DMEM (Cat. No. 11960-069) and fetal bovine serum (C at. N 0. SH 30070-03) were purchased from Invitrogen and Hyclone (Logan, UT, USA), respectively. Medium and supplements were combined according to manufacturer's instructions.

\section{Gene-expression analysis}

Total RNA was extracted directly from cells growing in six-well or 6-cm tissue culture plates using $Q$ iagen $R N$ easy mini kits according to the manufacturer's instructions. RNA concentrations were measured using a Beckman DU 530 or Nanodrop spectrophotometer and RNA quality determined by denaturing agarose gel electrophoresis or an Agilent 2100 bioanalyzer. Wholegenome expression analysis was carried out using Affymetrix Human Genome U 133 Plus $2.0 \mathrm{GeneC}$ hip $^{\circledR}$ system, Illumina 1 and Illumina 2, and RNA levels for certain genes were confirmed by qPCR. For Illumina BeadArrays, total RNA was linearly amplified and biotin-labeled using Illumina TotalPrep ${ }^{T M}$ kits (Ambion, TX, USA), and CRNA was quality controlled using an Agilent 2100 Bioanalyzer. cRNA was hybridized to Illumina BeadChips, 


\section{Figure 11. Proliferative potential of human embryonic progenitor cell lines.}

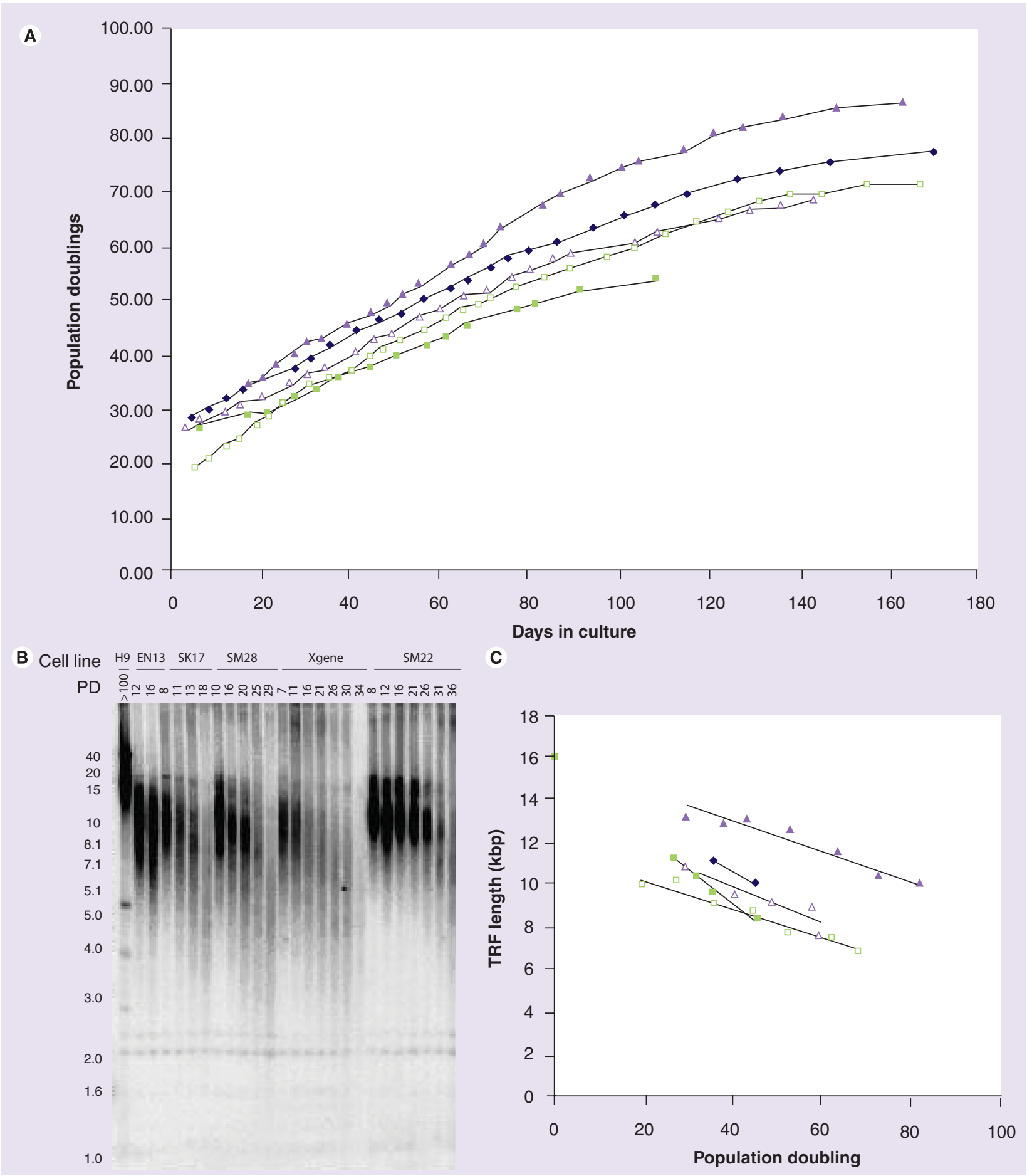

(A) Growth curves of the cell lines EN13 (filled diamond), SK17 (filled square), SM 28 (open triangle) and SM 22 (filled triangle), compared with neonatal foreskin fibroblasts (Xgene) (open square). (B) TRF analysis of DNA from human embryonic stem cells (H9), compared with the cell lines at various passage numbers; (C) Scatter plots of mean TRF length versus population doubling number.

PD: Population doubling; TRF: Terminal restriction fragment.

processed and read using a BeadStation array reader according to the manufacturer's instructions (Illumina). For Affymetrix GeneChip analysis, a two-cycle cRNA amplification and labeling was performed. A total of $100 \mathrm{ng}$ of total RNA from each sample was used for the 
first cycle of double-stranded cDNA synthesis using in vitro transcription amplification of CR N A (M EGAscript T 7 kit, Ambion,) followed by two cycles of target labeling (Affymetrix). Labeled CRNA $(15 \mu \mathrm{g})$ was fragmented and hybridized according to the manufacturer's instructions. RFU values for all of the cell lines with common probe sets were quantile normalized. In Figre3, variation of the levels of expression of a singlegene across cell lines was calculated as the ratio of the SD of RFU values/mean RFU and is reported as the SD/RFU ratio. In supplementary Tables $\mathbf{A 2}$, A3 \& A4, the genes are displayed in rank order (highest-lowest) for the ratio of highest RFU value observed for the gene in the entire set of cell lines/average RFU value. In supplementary TableA5, the top 45 differentially expressed genes were rank ordered (highest-lowest) for the ratio of highest RFU value observed for the gene in the individual cell line/average RFU value for all cell lines. In supplementary TadeA6, the genes corre sponding to recognized CD antigens are displayed in rank order (highest-lowest) and also (lowest-highest) for the ratio of highest RFU value observed for the gene in the entire set of cell lines/average RFU value and lowest RFU value observed for the gene in the entire set of cell lines/average RFU value, respectively. In supplementary TableA7, the genes corresponding to secreted proteins are displayed in rank order (highest-lowest) for the ratio of highest RFU value observed for the gene in the entire set of cell lines/average RFU value.

To validate the expression observed in BeadArray and GeneChip data sets, QPCR was used to independently measure RNA levels for FOXF1, FOXG1B, HOXA10, HOXA5, HOXB2, HOXB7, HOXB8, HOXB9, HOXC6, MYOD 1, MYOG, PRDX5, RPL24, SOX11, SOX4 and SOX8 genes in the cell lines cell lines B29, B30, E51, RAD 20-19, RAD 20-5, RAD 20-16, SK57, SK60, SK61, SK 17, SK 30, EN 31, W 4, W 10, SM 28, EN 5, EN 13, SK5, RASKEL6, RASKEL8, RASKEL 18, W 8, RAPEN D 17, E68, C 4ELS5-8, C4ELS5-6, E44, E3, EN 18, EN 47, E15, C4ELSR2, C4ELSR13 and EN 1. RNA used samples used for qPCR were the same as used for gene-expression analysis with the Illumina Beadchips or Affymetrix GeneC hips. The CDN A was synthesized with Invitrogen SuperScript III FirstStrand Synthesis SuperM ix for qRT-PCR and qPCR was performed using a Bio-Rad iCycler with an iQ 5 Multicolor Real-Time PCR D etection System. The reactions used I nvitrogen SYBR GreenER qPCR SuperM ix for the iCycler.
NMF consensus description

Gene-expression data were analyzed using N M F [43]. N M F is an unsupervised learning algorithm that identifies molecular patterns when applied to geneexpression data by detecting contextdependent patterns of gene expression in complex biological systems [44]. The N M F analysis was run in GenePattern, downloaded from the Broad Institute at $M$ assachusetts Institute of Technology $[45,101]$. T he parameters used for the N M F analysis shown in the NMF Consensus Plot (Figre10) were $\mathrm{N}=3232$ most differentially expressed gene and $M=202$ cell lines. N M F analyses were itera tively calculated with increasing $\mathrm{k}$ from 1 to 150 and selected a $\mathrm{k}=140(\mathrm{k}=142$, two samples not utilized in this report) based on stability of the calculated cophenetic coefficient to minimize the divergence norm. The default N M F Consensus settings of number of clusterings $=20$, number of iterations $=2000$, stop convergence $=40$ and stop frequency $=10$ [32].

\section{Tumorigenicity in mice}

Approximately 20 million cells from each of the cell lines B16, B28, 6-1, B26, B11, B2, CM 02, E75, E15, 4D 20.9, E72, EN 7, EN 55, SK 17 and Z11 were each injected into two SCID mice (or 10 million cells/mouse). $\mathrm{H}$ alf the cells (5 million) were injected intramuscularly into the right rear leg and the other 5 million subcutaneously into the left rear leg. After 4-6 months, each mouse was placed supine on the table, and under an operating microscope, bilateral skin incisions were made starting at the knee joint, and extending to the abdomen and then medially to the spine. The skin was then peeled back exposing all the surface leg muscles. The surface of the skin was examined, as well as the muscle surface. The muscles were transected every $2 \mathrm{~mm}$. The femur was exposed and examined. Following bilateral limb dissection and examination, the abdominal incision was extended anteriorly to the thymus gland, exposing all abdominal organs, tissues as well as the lungs and myocardium. Every organ and tissue (thymus gland, heart, lungs, kidneys, adrenal glands, liver, gastrointestinal organs, reproductive tract and the inner lining of the thoracic and abdominal cavity) were examined both on the surface and following transsection, under the operating microscope.

Flow cytometry analysis of cell

surface antigens

A representative number of cell lines at defined passage $(p)$ numbers (4D 20.8, p11; E68, p14; E109, p10; ELS5.8, p10; ELSR10, p15; M 10, 
p8; 7PEN D 24, p10; SK 17, p13) were analyzed by immunostaining for various cell surface antigens and flow cytometry analysis. Adherent cells were detached using ESG RO Complete Accutase (Chemicon/Millipore, Temecula, CA, USA) to minimize antigen degradation. $C$ ell aliquots were then incubated with the following standard panel of mouse monoclonal CD antibodies: CD24 (Chemicon; CBL561), CD 49b (Southern Biotech, Birmingham, AL, USA; 9426-01), CD 66a (R\&D Systems, M inneapolis, MN, USA; M AB2244), CD 81 (Santa Cruz Biotechnology, Santa Cruz, CA, U SA; SC-7637), CD 117 (Southern Biotech; 9816-01), CD 133 (Abcam, Cambridge, MA, USA; ab5558), CD 184 (BectonDickinson, San Jose, CA, USA; 555971), CD 252 (R\&D Systems; M AB 10541) at the manufacturers' recommended concentrations or at $10 \mu \mathrm{g} / \mathrm{ml}$, or an equivalent concentration of mouse isotype control IgG1, IgG 2a or IgG2b (Southern Biotech). The cells were then stained with Alexa Fluor 488-conjugated goat anti-mouse IgG $(\mathrm{H}+\mathrm{L})$ antibody (Invitrogen; A11029) and analyzed using a FACSC alibur flow cytometer (Becton-D ickinson) and FloJo software (TreeStar, Inc. Ashland, OR, USA).

\section{ELISA}

Cell culture medium from selected cell lines were quantitated for factors secreted into the medium utilizing the following ELISA or Duoset $(R \& D$ Systems) kits: AREG (Cat. N o. DY262, R\&D Systems), FGF-7/KGF (Cat. N o. DY251, R\& D Systems), IGFBP-5 (C at. N o. DY875, R\&D Systems), PDGF-BB (Cat. N o. DY220, R\&D Systems), TGF $\beta-1$ (Cat. No. DY240, R\&D Systems), TIM P-1 (Cat. N o. D Y970, R\&D Systems) and vitronectin (Cat. N o. TAK-M K102, Takara Bio distributed by Thermo Fisher Scientific, Waltham, MA, USA). The factors were quantitated in duplicate determinations.

Telomerase assays \& terminal restriction fragment analysis

TRAP assays were performed using a TRAPez ${ }^{\circledR}$ Kit (Chemicon). CHAPS lysates were prepared from cells and aliquots were frozen. Upon thawing, the lysates were subjected to protein quantification using the quick-start Bradford assay system (Biorad), and 26-cycle PCR-TRAPs were performed in linear range of the assay using $300 \mathrm{ng}$ of total protein lysate per reaction. TRAP products were resolved on $15 \%$ polyacrylamide large gels and exposed to phosphorimager screens. TRAP was performed as described previously. TRF length analysis was performed as described before [46]. In brief, genomic DN A was extracted from cells at different PD $s$ and subjected to restriction with $\mathrm{H}$ infl and Rsal and $2 \mu \mathrm{g}$ of the digested DNA was resolved on $0.5 \%$ agarose gels. The resulting denatured gels were directly incubated with a telomeric ${ }^{32} \mathrm{P}$-labeled $\left(\mathrm{C}_{3} \mathrm{TA}_{2}\right)_{3}$ probe. The dried gels were subsequently washed and exposed to phosphorimager screens for detection of the telomeric signal.

\section{Discussion}

We describe a simple combinatorial protocol that, similar to the shotgun cloning of genes, allows the nonspecific generation of a library of cell lines that can later be analyzed and collated using microarray and bioinformatics analysis. Surprisingly, many of the lines are capable of expansion in standard adherent culture and appear to display a wide array of markers of embryonic progenitor cell types from endodermal, mesodermal, ectodermal and neural crest lineages. The presence of diverse but discrete $\mathrm{H} O \mathrm{X}$ gene expression in these lines is consistent with the wide variety of $\mathrm{HOX}$ gene-expression patterns observed even in homologous cell types, such as dermal fibroblasts isolated from various regions of the body [11], and suggests that the clonal isolation may have occurred subsequent to the activation of these HOX genes, although the uniformity of these transcription factors in the clones was not assayed in this study. It should be noted that only a small field of combinations of differentiation conditions, differentiation times and subsequent clonal propagation medium were used in this study. Therefore, it is possible that further efforts to expand the conditions may yield additional cell types. It should also be noted that the variation of media used in propagating the lines may have been a source of variability in gene expression, and that to some degree the diversity observed may be due to the influence of the media, whereas the differentiated state of such cells would otherwise be identical. Further studies are warranted to study these effects.

A study of this scale required that individual assays, such as QPCR to confirm the microarray results, ELISA to measure immunoreactive secreted proteins and immunocytochemistry to confirm protein expression in situ or telomere assays, could only be performed on a small subset of the cell lines. Therefore, further study of the cell lines is required to interpret the gene-expression profiles reported. The ability to scale and cryopre serve many diverse hEP cell lines may allow the cells to bedistributed and thereby help standardize 
studies in stem cell biology. The robust proliferative capacity of many of the clones likely reflects the fact that they were recently isolated from $\mathrm{hES}$ cells that typically show germline telomere length (i.e., $15 \mathrm{kbp}$ TRF length). These unusually long telomeres give hEP cell lines a benefit compared with fetal- or adult-derived cells that typically have far shorter telomeres and because they are terminally differentiated often do not propagate in vitro. The scalability of hEP cell lines may therefore provide a useful point of scal ability other than the scaling of hES cell lines themselves. O ur initial profiling of hEP cell clones is necessarily limited and preliminary due to the large number of cell lines isolated and the fact that some of the cells were analyzed on the Affymetrix microarray platform and could not be normalized with the cell lines analyzed by Illumina microarrays. M any additional studies need to be performed on the differentiation potential and stability of the lines after being passaged in vitro. The presented data suggest that cloned libraries of hES-derived progenitor lines may provide a useful means of profiling the gene-expression profile of primitive cell types in order to identify their differentiation potential, for example, cell surface antigens,

\section{Executive summary}

The unique challenges of pluripotency

- Human embryonic stem (hES) cells show the capacity to differentiate into all of the hundreds of somatic cell lineages in the developing human.

- Human embryonic progenitor (hEP) cells are primitive precursors of terminally differentiated cells that are capable of propagation in vitro and display makers generally associated with the embryonic stages of development.

- Challenges to the field include the mapping of the 'embryome' for example to identify the unique molecular markers that allow the identification and isolation of hEP cells.

Multiplex generation \& characterization of hEP cell clones

- The ACTCellerate protocol utilizes a two-step differentiation and propagation protocol to isolate clonal populations of scalable hEP cell lines. Of 1090 clones isolated in this report, 280 lines $(25.7 \%)$ expanded to at least four roller bottles.

Clonal hEP cells do not display hES markers but instead show markers of diverse primitive embryonic progenitors

- Cell lines derived with the ACTCellerate protocol do not express markers of hES cells such as hTERT or OCT4.

- A total of $71 \%$ of the hEP cell lines show positive expression for MEOX1, MEOX2, or FOXF1, which in the mouse are reported to be expressed only in early stages of embryonic development.

- Non-negative matrix factorization suggested that the complexity of distinct cell types isolated was at least 140.

Immunocytochemical confirmation of hEP microarray gene-expression analysis

- Selected markers in putative ectodermal, mesodermal, endodermal and neural crest gene cell lines were confirmed by immunocytochemistry.

\section{Clonal hEP lines express diverse cell surface antigens}

- Flow cytometry confirmed that gene expression often predicts CD antigen expression on the cell surface and provides a means of manipulating hEP cell types.

\section{hEP clones express unique secreted factors}

- hEP cell clones expressed diverse secreted growth factors. Select factors confirmed by ELISA included AREG, FGF7, IGFBP5, TGF $\beta$-1 and PDGF-BB.

\section{hEP cells lack tumorigenicity}

- hEP cell lines expressed a wide array of oncofetal genes including: SILV, PLAG1, AMIGO2, HCLS1, SPINK1, PRAME, INSM1, ENC1 and CEACAM1, as well as others.

- Clonal hEP cell lines did not generate malignant tumors in 4-6 months at doses of 10 million cells per mouse when injected subcutaneously and intramuscularly.

\section{hEP cells include clones with a robust \& mortal proliferative capacity}

- hES cells express high levels of telomerase activity by telomeric repeat amplification protocol (TRAP) assay, and an immortal phenotype when maintained in the undifferentiated state.

- hEP cells were telomerase negative by TRAP assay, but often display a long proliferative lifespan in vitro useful in scaling the cells. 
including growth factor receptors, and secreted proteins, such as growth factors and cytokines. The potential of such cells for use in therapy awaits definition of the developmental potential of the cell lines and studies of the survival and function of such primitive cells in normal or pathological adult tissue (heterochronic transplantation). Because these lines could easily be documented by photomicroscopy to have a differentiated morphology when originally plated as a single cell, clonal propagation may provide a useful means of insuring the absence of contaminating hES cells in formulations or other cell types that could lead to tumor formation or the differentiation of undesired cell types.

The prospect of generating larger libraries of hEP cell clones and the complex and poorly characterized markers for early human embryonic lineages, with a complexity that likely exceeds $10^{3}$, highlights the need to database the markers and cell surface antigens of the early lineages of the human developmental tree [47]. Such a database and a large library of defined cell lines may facilitate the translation of the developmental potential of hES cells into actual cell therapies.

\section{Acknowledgements}

We thank Warren H oeffler of Xgene Corporation for the use of the foreskin fibroblast line des gnated Xgene, Jill Cheng of Novartis for consulting on the analysis of the microarray data, Kenneth Beckman (Children's Hospital Oakland Research Institute, 0 akland, CA, U SA) for assistance in performing the Illumina microarray analysis, and M Bigos and $\checkmark$ Stepps (Gladstone Institute/U niversity of California San Francisco Flow Cytometry Core facility, U SA) for assistance with FACS analysis.

Financial \& competing interests disclosure MD West, J Sampathkumar and J Long are employees (M W) or consultants (JS and JL) of BioTime, Inc., and R G eoffrey Sargent, C Brown, C Burrows, J Song Chu, S Kessler, D Larocca and J M urai are employees of Advanced Cell Technology, Inc., both companies operating in the field of regenerative medicine.

The authors have no other relevant affiliations or financial involvement with any organization or entity with a financial interest in or financial conflict with the subject matter or materials discussed in the manuscript apart from those disd osed.

No writing assistance was utilized in the production of this manuscript.

\section{Bibliography}

1. Thomson JA, Itskovitz-Eldor J, Shapiro SS et al.: Embryonic stem cell lines derived from human blastocysts. Science 282 , 1145-1147 (1998).

2. Shamblott M J, Axelman J, Wang $S$ et al.: D erivation of pluripotent stem cells from cultured human primordial germ cells. Proc. N atl Acad. Sci. U SA 95, 13726-13731 (1998).

3. Blentic A, Tandon P, Payton S et al.: The emergence of ectomesenchyme. Dev. Dyn. 237(3), 592-601 (2008).

4. Carletti B, Grimaldi P, M agrassi L, Rossi F: Specification of cerebellar progenitors after heterotopic-heterochronic transplantation to the embryonic CN S in vivo and in vitro. J. N eurosci. 22, 7132-7146 (2002).

5. M inguet $S, C$ ortegano I, Gonzalo $P$ et al.: A population of C-Kit(low) (CD 45/TER 119)-hepatic cell progenitors of 11-day postcoitus mouse embryo liver reconstitutes cell-depleted liver organoids. J. Clin. Invest. 112, 1152-1163 (2003).

6. Quackenbush EJ, Wershil BK, Aguirre V, Gutierrez-Ramos JC: Eotaxin modulates myelopoiesis and mast cell development from embryonic hematopoietic progenitors. Blood 92, 1887-1897 (1998).

7. Kitisin K, Saha T, BlakeT et al.: TGF- $\beta$ signaling in development. Sci. ST KE 2007(399), cm1 (2007).
8. Correia AS, Anisimov SV, Li JY, Brundin P: Stem cell-based therapy for Parkinson's disease. Ann. M ed. 37, 487-498 (2005).

9. Abranches $E$, Bekman $E, H$ enrique D, Cabral JM : Expansion of mouse embryonic stem cells on microcarriers. Biotechnol. Bioeng. 96, 1211-1221 (2007).

10. Smith JR, Pereira-Smith O M, Schneider EL: Colony size distributions as a measure of in vivo and in vitro aging. Proc. $\mathrm{N}$ atl Acad. Sci. U SA 75, 1353-1356 (1978).

11. Rinn JL, Bondre C, G ladstone H B, Brown PO, Chang HY: Anatomic demarcation by positional variation in fibroblast gene expression programs. PLOS Genet. 2, el19 (2006).

12. M cGinnis W, Krumlauf $R$ : $H$ omeobox genes and axial patterning. Cell 68, 283-302 (1992).

13. Candia AF, $\mathrm{Hu} \mathrm{J}, \mathrm{Crosby} J$ et al.: M ox-1 and M ox-2 define a novel homeobox gene subfamily and are differentially expressed during early mesodermal patterning in mouse embryos. D evelopment 116, 1123-1136 (1992).

14. Candia AF, Wright CV: D ifferential localization of M OX-1 and M ox-2 proteins indicates distinct roles during development. Int. J. D ev. Biol. 40, 1179-1184 (1996).
15. Aitola M, Carlsson P, M ahlapuu M , Enerback S, Pelto-H uikko $M$ : Forkhead transcription factor FoxF2 is expressed in mesodermal tissues involved in epithelio-mesenchymal interactions. D ev. D yn. 218, 136-149 (2000).

16. Sasaki H, H ogan BL: D ifferential expression of multiple fork head related genes during gastrulation and axial pattern formation in the mouse embryo. D evelopment 118, 47-59 (1993).

17. Kim TA, Jiang $S$, Seng $S$, Cha K, Avraham H K, Avraham S: The BT B domain of the nuclear matrix protein N RP/B is required for neurite outgrowth. J. Cell Sci. 118, 5537-5548 (2005)

18. Kim TA, Lim J, $O$ ta S et al.: N RP/B, a novel nuclear matrix protein, associates with p110(RB) and is involved in neuronal differentiation. J. Cell Biol. 141, 553-566 (1998).

19. Kitanaka J, Takemura M, M atsumoto K, M ori T, Wanaka A: Structure and chromosomal localization of a murine LIM /homeobox gene, Lhx8. Genomics 49, 307-309 (1998).

20. Al-Shawi R, Ashton SV, Underwood C, SimonsJP: Expression of the Ror1 and Ror2 receptor tyrosine kinase genes during mouse development. D ev. Genes Evol. 211, 161-171 (2001). 
21. Blaschke RJ, M onaghan AP, Schiller $\mathrm{S}$ et al.: SH OT, a SH OX-related homeobox gene, is implicated in craniofacial, brain, heart, and limb development. Proc. N atl Acad. Sci. U SA 95, 2406-2411 (1998).

22. Stipp CS, Litwack ED, Lander AD: Cerebroglycan: an integral membrane heparan sulfate proteoglycan that is uniqueto the developing nervous system and expressed specifically during neuronal differentiation. J. Cell Biol. 124, 149-160 (1994).

23. Ribes V, Fraulob V, Petkovich M, D olle P: The oxidizing enzyme CYP26al tightly regulates the availability of retinoic acid in the gastrulating mouse embryo to ensure proper head development and vasculogenesis. Dev. D yn. 236, 644-653 (2007).

24. Besser $D$ : Expression of nodal, lefty-a, and lefty- $B$ in undifferentiated human embryonic stem cells requires activation of Smad2/3. J. Biol. Chem. 279, 45076-45084 (2004).

25. Wang ZX, Kueh JL, Teh $\mathrm{CH}$ et al.: Zfp206 is a transcription factor that controls pluripotency of embryonic stem cells. Stem Cells 25, 2173-2182 (2007).

26. Lim LS, Loh YH , Zhang $W$ et al.: Zic3 is required for maintenance of pluripotency in embryonic stem cells. M ol. Biol. Cell 18, 1348-1358 (2007).

27. Thuret $S, B$ hatt $L, O$ 'Leary $D D$, Simon $\mathrm{H} \mathrm{H}$ : Identification and developmental analysis of genes expressed by dopaminergic neurons of the substantia nigra pars compacta. M ol. Cell. N eurosci. 25, 394-405 (2004).

28. Roubin R, Pizette $S$, O llendorff $V$, Planche J, Birnbaum D, D elapeyriere 0 : Structure and developmental expression of mouse $G$ arp, a gene encoding a new leucinerich repeat-containing protein. Int. J. D ev. Biol. 40, 545-555 (1996).

29. H idai $C$, Zupancic $T$, Penta $K$ et al.: Cloning and characterization of developmental endothelial locus-1: an embryonic endothelial cell protein that binds the $\alpha \mathrm{V} \beta 3$ integrin receptor. Genes D ev. 12, 21-33 (1998).
30. Koch M, Laub F, Zhou P et al.: Collagen XXIV, a vertebrate fibrillar collagen with structural features of invertebrate collagens: selective expression in developing cornea and bone. J. Biol. Chem. 278, 43236-43244 (2003).

31. Goldberg JL, Vargas ME, Wang JT et al.: An oligodendrocyte lineage-specific semaphorin, Sema5A, inhibits axon growth by retinal ganglion cells. J. N eurosci. 24, 4989-4999 (2004).

32. D ahlstrand J, Lardelli M, Lendahl U : $N$ estin mRN A expression correlates with the central nervous system progenitor cell state in many, but not all, regions of developing central nervous system. Brain Res Dev. Brain Res 84, 109-129 (1995).

33. Falk J, Bonnon C, Girault JA, Faivre-Sarrailh C: F3/contactin, a neuronal cell adhesion molecule implicated in axogenesis and myelination. Biol. Cell. 94, 327-334 (2002).

34. Besnard V, Wert SE, Kaestner KH, $W$ hitsett JA: Stage-specific regulation of Foxal and Foxa2 in mouse embryos and adult tissues. GeneExpr. Patterns5, 193-208 (2004).

35. Cereghini $\mathrm{S}, \mathrm{O}$ tt $\mathrm{M} \mathrm{O}$, Power $\mathrm{S}, \mathrm{M}$ aury $\mathrm{M}$ : Expression patterns of $\mathrm{nHNF}$ 1 and $\mathrm{HNF} 1$ homeoproteins in early postimplantation embryos suggest distinct and sequential developmental roles. D evelopment 116, 783-797 (1992).

36. Zhou J, Wang H, Lu A et al.: A novel gene, N M ES1, downregulated in human esophageal squamous cell carcinoma. Int. J. Cancer 101, 311-316 (2002).

37. M oll R, Simbelmann R, Goldschmidt M D et al.: The human gene encoding cytokeratin 20 and its expression during fetal development and in gastrointenstinal carcinomas. D ifferentiation 53, 75-93 (1993).

38. Karsch-M izrachi I, Travis M, Blau H Leinwand LA: Expression and DNA sequence analysis of a human embryonic skeletal muscle myosin heavy chain gene. N ucleic Acids Res. 17, 6167-6179 (1989).
39. Kachinsky AM, D ominov JA, M iller JB: Intermediate filaments in cardiac myogenesis: nestin in the developing mouse heart. J. H istochem. Cytochem. 43, 843-847 (1995).

40. Wagner $\mathrm{SN}$, Wagner $\mathrm{C}, \mathrm{H}$ ofler $\mathrm{H}$, Atkinson M J, Goos M : Expression cloning of the CD N A encoding a melanomaassociated $A g$ recognized by $m A b$ H M B-45. Identification as melanocytespecific Pmel 17 cD N A. Lab. Invest. 73, 229-235 (1995).

41. Baxter LL, Pavan WJ: Pmel17 expression is $M$ itf-dependent and reveals cranial melanoblast migration during murine development. Gene Expr. Patterns 3, 703-707 (2003).

42. Allsopp RC, Vaziri $\mathrm{H}$, Patterson $\mathrm{C}$ et al.: Telomere length predicts replicative capacity of human fibroblasts. Proc. $\mathrm{N}$ atl Acad. Sci. U SA 89, 10114-10118 (1992).

43. Lee D D, Seung H S: Learning the parts of objects by non-negative matrix factorization. Nature 401, 788-791 (1999).

44. Brunet JP, Tamayo P, Golub TR, $M$ esirov JP: $M$ etagenes and molecular pattern discovery using matrix factorization. Proc. N atl Acad. Sci. U SA 101, 4164-4169 (2004).

45. Reich M, Liefeld T, Gould J, Lerner J, Tamayo P, M esirov JP: G enePattern 2.0. $N$ at. Genet. 38, 500-501 (2006).

46. Vaziri $H$, West M D, Allsopp RC et al.: AT M -dependent telomere loss in aging human diploid fibroblasts and D N A damage lead to the post-translational activation of $\mathrm{p} 53$ protein involving poly(AD P-ribose) polymerase. EM BO J. 16, 6018-6033 (1997)

47. West M D, M ason $C$ : $M$ apping the human embryome: 1 to $10 \mathrm{el} 3$ and all the cells in between. Regen. M ed. 2, 329-333 (2007).

Website

101. GenePattern

www.broad.mit.edu/cancer/software/ genepattern 